\title{
Translational motion compensation for ISAR imaging under low SNR by minimum entropy
}

\author{
Lei Zhang ${ }^{1 *}$, Jia-lian Sheng ${ }^{1}$, Jia Duan', Meng-dao Xing ${ }^{1}$, Zhi-jun Qiao ${ }^{2}$ and Zheng Bao
}

\begin{abstract}
In general, conventional error correction for inverse synthetic aperture radarimaging consists of range alignment and phase adjustment, which compensate range shift and phase error, respectively. Minimum entropy-based methods have been proposed to realize range alignment and phase adjustment. However, it becomes challenging to align high-resolution profiles when strong noise presents, even using entropy minimization. Consequently, the subsequent phase adjustment fails to correct phase errors. In this article, we propose a novel method for translational motion correction, where entropy minimization is utilized to achieve range alignment and phase adjustment jointly. And, a coordinate descent algorithm is proposed to solve the optimization implemented by quasi-Newton algorithm. Moreover, a method for coarse motion estimation is also proposed for initialization in solving the optimization. Both simulated and real-measured datasets are used to confirm the effectiveness of the joint motion correction in low signal-to-noise ratio situations.
\end{abstract}

Keywords: Inverse synthetic aperture radar (ISAR), Motion compensation, Low signal-to-noise ratio (low SNR), Range alignment, Phase adjustment, Minimum entropy

\section{Introduction}

Inverse synthetic aperture radar (ISAR) imaging has been a widely addressed topic in last few decades [1-3]. In order to achieve high-resolution both in range and cross-range of target imagery, the ISAR imaging technique exploits both wideband characteristics of radar waveform and the diversity of viewing aspect angle from radar to the target. In general, the range resolution is proportional to bandwidth of waveform, and the crossrange resolution is dependent on both the coherent processing interval (CPI) and the target rotational motion from variation of radar viewing angles. Therefore, CPI should be long enough to achieve high cross-range resolution by Doppler analysis. In ISAR scenarios, the target is often engaged in complicated maneuvers and the translational motion should be compensated before performing imaging processing. Translational motion introduces range misalignment and high-order phase error. For ISAR imaging of a non-cooperative target, a data-driven compensation procedure must be accounted,

\footnotetext{
* Correspondence: leizhang@xidian.edu.cn

${ }^{1}$ National Key lab of Radar Signal Processing, Xidian University, Xi'an 710071, P. R. China

Full list of author information is available at the end of the article
}

which generally consists of range alignment and phase adjustment.

Range alignment is to compensate the range shifts of profiles. Without priorknowledge available about the range shifts, range alignment is usually realized based on the similarity of high-resolution range profiles (HRRPs). Typical methods for range alignment can be sorted into three groups. The first class is based on a maximum correlation between adjacent profiles [4]. The dominant scatter method tracks a prominent scatter and estimates the range shift. And, the maximum correlation method [4] aligns each HRRP by using the principle that the envelope correlation of two adjacent profiles reaches a maximum when they are aligned. Actually, the principle of maximum correlation of two adjacent profiles can be regarded as a local optimization of alignment. Because the estimation of range shifts between every two adjacent envelopes is independent of each other. As a result, this method is sensitive to target scintillation. And, it is likely to fail when strong noise presents, as the coherence of HRRPs is contaminated seriously. The other group is optimization-based methods [5-9]. It is widely accepted that global optimization methods are more robust to reflectivity scintillation and additive noise than 
maximum correlation method, in which the problem of ISAR range alignment is formulated by using some global metrics. Minimum entropy or maximum contrast of the synthetic profile, such as the average range profile (ARP) of the aligned profiles, is used as the criterion to evaluate the performance of range alignment. The synthetic profile usually has the highest sharpness when the profiles are aligned perfectly. Or else, the sharpness of synthetic envelope reduces. It is reasonable to evaluate the sharpness of the synthetic profile by contrast or entropy and establish an optimization for range alignment. In general, the synthetic profile is calculated as the mean of magnitude of the aligned HRRPs. Therefore, the synthetic profile can be viewed as a non-coherent energy accumulation of aligned profiles, and it can overcome the noise interference in some degree. However, in the situations of low signal-to-noise ratio (SNR), the SNR gain from the non-coherent integrant is not enough to overcome the interference from noise on the synthetic profile, leading to that the consistency between the sharpness of the synthetic profile and the quality of range alignment is broken. In this sense, it is still challenging to achieve optimal range alignment in presence of strong noise.

If range alignment is done well, afterwards, phase adjustment is carried out to remove the error phase. There are many different schemes to perform phase adjustment, which can be sorted into different groups. The first is based on tracking a phase of dominant scattering centers. Phase gradient autofocus algorithm [10], the multiple dominant scatters algorithm [11], and the weighted least square phase estimation [12] can be classified into this group. These methods usually perform well when dominant scattering centers can be extracted from HRRPs. However, presence of strong noise brings inherent difficulty to precise phase tracking through several dominant scatters. Another group numerically optimizes the phase error correction to improve a global metric consistent with image focus, in which image contrast (IC) [13-16] and entropy [17-22] are utilized as the cost function to optimize the phase error. Image metricbased approaches are usually able to obtain an optimal solution even in the presence of strong background noise and clutter. However, successful phase adjustment can only be ensured when perfect range alignment is obtained, while if the profiles are misaligned in some cases, such as strong noise situation, even the image metric-based methods fail to correct phase errors. To overcome this problem, in [14], a joint correction scheme for simultaneous range alignment and phase adjustment was proposed based on a two-order polynomial model of the translational range history, and a novel coarse estimation of both velocity and acceleration was also developed to accelerate the motion estimation. In the issue of ISAR motion compensation, adaptive joint time-frequency algorithm (AJTFA) also plays a significant role in ISAR motion compensation and imaging processing [11,23,24]. Especially, AJTFA is inherently suitable to dealing with maneuvering targets by projecting the one-dimensional signal onto the twodimensional (2D) time-frequency plane, and the motion parameters can be extracted from the time-frequency spectrum of dominant scatters. However, strong noise can easily submerge the time-frequency spectrum degrading its performance in low SNR scenarios.

In radar remote sensing, strong noise usually presents, due to the signal decay from long range and absorption of transmit medium. The SNR problem is among the most significant challenges that ISAR imaging systems frequently face. In the presence of strong noise, motion compensation for ISAR imaging inherently encounters some difficulties. Therefore, the technique of motion compensation under low SNR is important, which may furnish imaging capability and improve the effective operating range of some ISAR systems with low power. Based on the fact that both range shift and phase error are directly related to the quality of the focused image, in this article, we present a novel entropy-based approach to joint range alignment and phase adjustment. It should be emphasized that the idea of joint correction of range shift and phase error has been proposed in [14], which motivates this study. In the joint correction, instead of separating motion compensation into the two dependent steps, it estimates the range shift and phase error simultaneously. Therefore, high SNR gain from 2D coherent integrant is benefited by both range alignment and phase adjustment.

In this article, the joint correction with entropy minimization models the translational motion as a highorder polynomial function [25], and 2D image entropy is minimized to optimize the polynomial coefficients. A novel coordinate descent algorithm is proposed to solve the minimum entropy optimization. The coordinate descent algorithm is implemented by the quasi-Newton algorithm, yielding fast convergence. For an optimization problem, the initialization is usually important to the efficiency and precision of the solutions. In this article, we also propose a method to estimate a coarse motion, which is effective to obtain the coarse coefficients efficiently. They can be applied as additional information to accelerate and promote the coordinate descent estimation. By using real datasets, the effectiveness of the joint motion compensation is validated.

The organization of this article is as follows. In "Signal model and minimum entropy compensation methods" section, we introduce the signal model and recall some existing minimum entropy-based methods for translational motion correction. In "Joint range alignment and phase adjustment by minimum entropy" section, algorithm for joint range alignment and phase correction by 
minimum entropy is illustrated in detail. Experimental results from both synthetic and real data processing are given in "Performance analysis" section to illuminate the effectiveness of the proposal. In the last section, some conclusions are drawn and some future works are viewed.

\section{Signal model and minimum entropy compensation methods}

\subsection{Signal model}

By referring to [14], a three-dimensional geometry is developed as shown in Figure 1, where the system of coordinates is denoted by $\left(\chi_{1}, \chi_{2}, \chi_{3}\right)$. And, the target is moving along an arbitrary trajectory together with angular motions. During a short dwell time, the effective angular motion can be assumed constant, representing by the effective rotational velocity $\omega_{\text {eff }}$ (whose direction is orthogonal to the line of radar sight). In this case, the effective angular motion of the target is restricted within a plane orthogonal to the effective rotational velocity. As a result, the geometry can be simplified into a $2 \mathrm{D}$ model [17] as Figure $1 \mathrm{~b}$ presents. In the $2 \mathrm{D}$ geometry, target motion is divided into translation and rotation as shown in Figure 1. And, translation is directly corresponding to the radial range $R(t)$ from the target center to the radar. The scattering field of the target is denoted as $L$. And, the instantaneous rotational angle of target is defined as $\theta(t)=\omega_{\text {eff }} t$, and $t$ is the slow time with $0 \leq t \leq T_{a}$, where $T_{a}$ is the CPI. The instantaneous range from scattering center at $(x, y)$ to radar is given by

$$
r(t)=R(t)+x \sin \theta(t)-y \cos \theta(t)
$$

where $R(t)$ denotes instantaneous distance from radar to the rotation center, usually representing the translational motion. $\theta(t)$ is the instantaneous rotation angle. Assuming the radar transmits a chirp waveform that

$$
s_{T}(\tau)=\operatorname{rect}\left(\frac{\tau}{T_{p}}\right) \cdot \exp \left(j \pi \gamma \tau^{2}\right)
$$

where $\tau$ denotes the fast time, $T_{\mathrm{p}}$ is the pulse duration, and $\gamma$ is the chirp rate. Therefore, the received signal from the target after down-conversion to the base-band is given by

$$
s_{R}(\tau, t)=\iint_{(x, y) \in L} A(x, y) \cdot s_{T}\left[\tau-\frac{2 r(t)}{c}\right] \cdot \exp \left[-j 2 \pi f_{c} \frac{2 r(t)}{c}\right] d x d y
$$

where $A(x, y)$ denotes the scattering coefficient at $(x, y)$ and $c$ is the light speed and the carrier frequency is $f_{\mathrm{c}}$. By applying Fourier transform with respect to $\tau$ and neglecting the introduced constants, we have

$$
s_{F}\left(f_{r}, t\right)=\iint_{(x, y) \in L} A(x, y) \cdot s_{T}\left(f_{r}\right) \cdot \exp \left[-j 2 \pi \frac{2 r(t)}{c}\left(f_{r}+f_{c}\right)\right] d x d y
$$

where $s_{T}\left(f_{r}\right) \approx \operatorname{rect}\left(\frac{f_{r}}{T_{p} \gamma}\right) \cdot \exp \left(j \pi \frac{f_{r}^{2}}{\gamma}\right)$ is the Fourier transform of $s_{T}(\tau)$ [26]. Applying the matched-filtering to $s\left(f_{r}\right.$, $t)$ by multiplying with $s_{T}^{*}\left(f_{r}\right)\left(s_{T}^{*}\left(f_{r}\right)\right.$ denotes the conjugate of $\left.s_{T}\left(f_{r}\right)\right)$ and omitting the introduced constant, the signal is expressed as

$$
\begin{aligned}
s\left(f_{r}, t\right)= & \iint_{(x, y) \in L} A(x, y) \cdot \exp \left[-j 2 \pi \frac{2 r(t)}{c}\left(f_{r}+f_{c}\right)\right] d x d y \\
= & \iint_{(x, y) \in L} A(x, y) \cdot \exp \left[-j 4 \pi \frac{x \sin \theta(t)-y \cos \theta(t)}{c}\left(f_{r}+f_{c}\right)\right] \\
& \cdot \exp \left[-j 2 \pi \frac{2 R(t)}{c}\left(f_{r}+f_{c}\right)\right] d x d y
\end{aligned}
$$

Assuming the effective rotational velocity during the CPI is $\omega_{\text {eff }}$, we have the rotation angle $\theta(t)=\omega_{\text {eff }} t$. In general, the rotation angle during CPI for ISAR imaging is very small, such as $3-4^{\circ}$. Therefore, we have the approximation that

$$
\left\{\begin{array}{c}
\cos \theta(t) \approx 1 \\
\sin \theta(t) \approx \omega_{\text {eff }} \cdot t
\end{array}\right.
$$

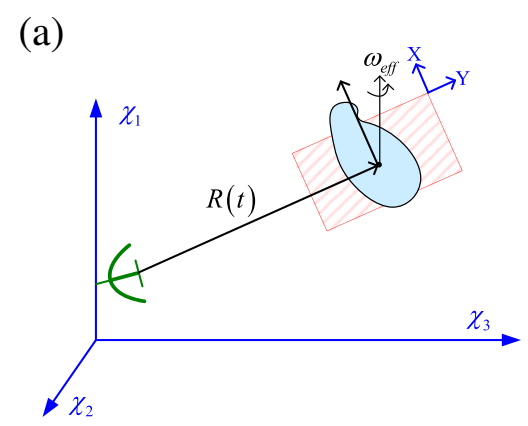

(b)

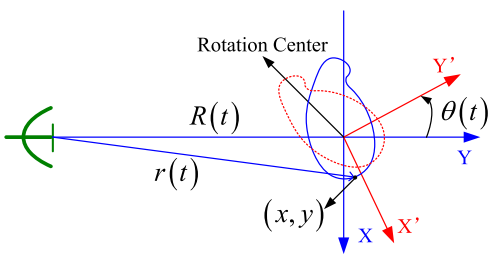

Figure 1 ISAR geometry. (a) Three-dimensional geometry. (b) Simplified 2D geometry. 
Based on this approximation, we have

$$
\begin{array}{r}
s\left(f_{r}, t\right) \approx \iint_{(x, y) \in L} A(x, y) \cdot \exp \left[-j 4 \pi \frac{x \omega_{\mathrm{eff}} t-y}{c}\left(f_{r}+f_{c}\right)\right] \\
\cdot \exp \left[-j 2 \pi \frac{2 R(t)}{c}\left(f_{r}+f_{c}\right)\right] d x d y
\end{array}
$$

In general, during a short dwell-time the range cell migration (RCM) induced by rotation can be restricted within a range cell. Otherwise, this RCM should be compensated by the keystone transform-based method $[27,28]$. Keystone transform is capable of removing the linear RCM effectively by removing the first-order coupling term in the frequency domain. And, it can be implemented in a very efficient way. Assuming the RCM caused by angular motion is corrected, we have

$$
\begin{array}{r}
s\left(f_{r}, t\right) \approx \iint_{(x, y) \in L} A(x, y) \cdot \exp \left[-j 4 \pi \frac{x \omega_{\mathrm{eff}} t-y}{c}\left(f_{r}+f_{c}\right)\right] \\
\cdot \exp \left[-j 2 \pi \frac{2 R(t)}{c}\left(f_{r}+f_{c}\right)\right] d x d y
\end{array}
$$

In (8), the phase term $\exp \left[-j 2 \pi \frac{2 R(t)}{c} f_{r}\right]$ causes misalignment of range profiles in the range-compressed domain. And, the term $\exp \left[-j 2 \pi \frac{2 R(t)}{c} f_{c}\right]$ stands for the phase error. Unless an optimal translational motion compensation is obtained, serious blurring can result in the ISAR image formed by the inversed Fourier transform, which assumes that the scatter behaves with only the linear phase $\exp \left(-j 4 \pi \frac{x \omega_{e f f} t}{\lambda}\right)$. It should be noted that compensations of the range misalignment and the phase error require different precisions. To remove the profile migration through range cells, the precision of the range shift should be in the order of a fraction of a range cell, such as a quarter of one range cell, which is typically tens of centimeters. However, the phase error corresponds to the radar operating wavelength, which is typically only a few centimeters. Limited by the range resolution, the motion estimate from range alignment is insufficient to perform an optimal phase correction. Moreover, due to the shortness of wavelength, the phase errors are largely wrapped, resulting in the uncertainty between the phase error and $R(t)$. Therefore, translational motion compensation typically consists of two steps: range alignment and phase adjustment [29]. And, the range alignment is usually viewed as the coarse compensation and phase adjustment as fine compensation. For clarity, $s\left(f_{n}, t\right)$ is rewritten by

$$
\begin{aligned}
s\left(f_{r}, t\right) \approx \iint_{(x, y) \in L} A(x, y) & \cdot \exp \left(-j 4 \pi \frac{x \omega_{\mathrm{eff}} t}{\lambda}\right) \cdot \exp \left(j 4 \pi \frac{y}{c} f_{r}\right) \\
\cdot & \exp \left[-j 2 \pi \cdot \Delta t_{e}(t) \cdot f_{r}\right] \\
\cdot & \exp [-j \Delta \phi(t)] d x d y
\end{aligned}
$$

In (9), the range migration and phase error are represented by two independent variants, denoted by $\Delta t_{e}(t)=\frac{2 R(t)}{c}$ (range shift) and $\Delta \phi(t)=2 \pi \frac{2 R(t)}{\lambda}$ (phase error), respectively. The phase in (9) is separated into four parts: the first is the linear phase term corresponding to the Doppler, the second term corresponds to the range compression, the third term corresponds to the range migration, and the last phase term is the phase error from translational motion. Considering the inevitable noise, the echoed signal is expressed in a discrete form as

$$
\begin{array}{r}
s(m, n)=\widetilde{s}(m, n) \cdot \exp \left[-j 2 \pi \cdot \Delta t_{e}(n) \cdot\left(m \cdot \Delta f_{r}\right)\right] \\
\cdot \exp [-j \Delta \phi(n)]+\varepsilon_{F}(m, n)
\end{array}
$$

And, by neglecting the constant terms, we have

$$
\begin{aligned}
\widetilde{s}(m, n) & =\iint_{(x, y) \in L} A(x, y) \cdot \exp \left[-j 4 \pi \frac{y}{c} \cdot\left(m \cdot \Delta f_{r}\right)\right] \\
f_{d} & =2 \pi \frac{x \omega}{\lambda}
\end{aligned}
$$

where $\varepsilon_{F}(m, n)$ denotes the complex noise, and $\Delta t_{e}(n)=$ $\frac{2 R(n)}{c}$ and $\Delta \phi(n)=4 \pi \frac{R(n)}{\lambda}$ are the range migration and phase error corresponding to the $n$th pulse, which are expected to be corrected in the range alignment and phase adjustment, respectively.

\subsection{Related works}

We focus on motion compensation for ISAR imaging under low SNR, which has been gained noticeable interests till now. To overcome the interference of strong noise and clutter, current methods are usually following a similar principle, in which, estimation of errors is converted into a problem of minimizing (or maximizing) a cost function. And, the cost function is selected such that when it is minimized (or maximized), optimal focusing is achieved. Entropy is one of the most primary function in error compensation for ISAR imaging. Entropy of 2D image (or one-dimensional synthetic range profile) represents its sharpness, and generally the "sharpest" image is corresponding to the fully focused image (or aligned HRRPs). Before presenting our method, herein, we introduce two typical 
entropy-based methods for range alignment and phase adjustment, respectively.

\subsubsection{Minimum entropy for range alignment (MERA)}

Approaches to range alignment by minimum entropy of synthetic range profile are discussed in [6-8]. Herein, we introduce them briefly. Applying inversed discrete Fourier transform (IDFT) to $s(m, n)$ and taking the magnitude, the expression of range profile after compensating the range shift by $t_{e}$ is given by

$$
\begin{aligned}
p\left(k, n ; t_{e}\right)= & a b s\left\{\frac{1}{M} \sum_{m=0}^{M-1} \exp \left(j 2 \pi \frac{k m}{M}\right) \cdot s(m, n)\right. \\
& \left.\cdot \exp \left[-j 2 \pi \cdot t_{e}(n) \cdot\left(m \cdot \Delta f_{r}\right)\right]\right\} k=0,1, \ldots, M-1 \\
= & a b s\left\{\frac{1}{M} \sum_{m=0}^{M-1} \exp \left(j 2 \pi \frac{k m}{M}\right) \cdot \widetilde{s}(m, n)\right. \\
& \left.\cdot \exp \left[j 2 \pi \cdot\left(\Delta t_{e}(n)-t_{e}(n)\right) \cdot\left(m \cdot \Delta f_{r}\right)\right]+\varepsilon(k, n)\right\}
\end{aligned}
$$

where abs[. ] is the operator for magnitude. And, symbol $\varepsilon(k, n)$ denotes the complex noise after IDFT. For simplicity, we do not write $\varepsilon(k, n)$ as a function of $t_{e}$ since range shift doesnot change the random statistics of noise. It is notable that noise is coupled in the range profile and distorts the range profile of target with increase of its energy. And, the ARP after compensating the $t_{e}$ is written as

$$
p_{\mathrm{APR}}\left(k ; t_{e}\right)=\frac{1}{N} \sum_{n=0}^{N-1} p\left(k, n ; t_{e}\right)
$$

The averaging processing is in some sense of noncoherent accumulation, and the distortion from noise can be suppressed by some degree. However, due to that no phase information is utilized, this non-coherent processing generally provides limited SNR gain compared to coherent integral. The entropy of ARP is a function of $t_{e}$, which is given by

$$
E_{\mathrm{APR}}\left(t_{e}\right)=-\frac{1}{S_{\mathrm{APR}}} \sum_{k=0}^{M-1}\left|p_{\mathrm{APR}}\left(k, t_{e}\right)\right|^{2} \ln \left|p_{\mathrm{APR}}\left(k, t_{e}\right)\right|^{2}+\ln S_{\mathrm{APR}}
$$

The density of ARP is defined as

$$
E_{\mathrm{APR}}\left(t_{e}\right)=-\frac{1}{S_{\mathrm{APR}}} \sum_{k=0}^{M-1}\left|p_{\mathrm{APR}}\left(k, t_{e}\right)\right|^{2} \ln \left|p_{\mathrm{APR}}\left(k, t_{e}\right)\right|^{2}+\ln S_{\mathrm{APR}}
$$

The optimal solution of range shift is estimated by minimizing the entropy of the synthetic profile.

$$
\left\langle\hat{t}_{e}\right\rangle=\underset{t_{e}}{\arg \min } E_{\mathrm{APR}}\left(t_{e}\right)
$$

In order to solve the optimization problem in (16), many searching approaches have been proposed by adopting exhaustive search or iterative search.

\subsubsection{Minimum entropy for phase adjustment (MEPA)}

Phase adjustment via minimum entropy is widely applied in issues of radar imaging [17-22]. It usually performs well in presence of strong noise and clutter, and it has no constraints on the error form. In minimum entropy phase correction, the range shift is assumed to be corrected, and then the entropy of $2 \mathrm{D}$ image is utilized as the cost function of phase error. The complex-valued HRRP after phase error compensation is expressed as

$$
\begin{aligned}
s_{c}(k, n)= & \frac{1}{M} \sum_{m=0}^{M-1} \exp \left(j 2 \pi \frac{k m}{M}\right) \cdot \widetilde{s}(m, n) \\
& \cdot \exp \{j \cdot[\phi(n)-\Delta \phi(n)]\}+\varepsilon(k, n)
\end{aligned}
$$

where $\phi$ denotes the compensated phase error. And, the complex-valued 2D image is obtained by applying IDFT to $s_{c}$ in cross-range direction, which is given by

$$
g(k, h ; \phi)=\frac{1}{N} \sum_{n=0}^{N} \exp \left(j 2 \pi \frac{h n}{N}\right) \cdot s_{c}(k, n)
$$

The entropy of corrected image is defined as a function of $\phi$ and written as

$$
E_{g}(\phi)=-\frac{1}{S_{g}} \sum_{k=0}^{M-1} \sum_{h=0}^{N-1}|g(k, h ; \phi)|^{2} \ln |g(k, h ; \phi)|^{2}+\ln S_{g}
$$

The density of image is

$$
S_{g}=\sum_{k=0}^{M-1} \sum_{h=0}^{N-1}|g(k, h ; \phi)|^{2}
$$

It is performed to solve the following optimization to estimate the phase error.

$$
\langle\hat{\phi}\rangle=\underset{\phi}{\arg \min } E_{g}(\phi)
$$

Many searching approaches have been proposed to solve the minimization problem in Equation (21). Especially, three schemes are introduced in [19] in detail. Although they have different searching schemes and efficiencies, their performances are similar as the underlying principle is the same. In general, minimum entropy phase adjustment is implemented by an iterative solution, the image entropy decreases with the increase of 
iteration number until the estimate reaches an acceptable optimal convergence.

\section{Joint range alignment and phase adjustment by minimum entropy}

\subsection{Algorithm description}

In practice, due to the complex motion of the target and the variance of the system and the environment, the translational motion between the target and the radar usually has high-order terms. Without loss of generality, we model the translational motion error as a $K$-order polynomial, i.e.,

$$
R(n)=\sum_{k=1}^{K} \alpha_{k}(n \cdot \Delta t)^{k}
$$

where one notes that $k$ begins from 1 to $K$. The firstorder term is corresponding to the velocity. Velocity only induces an additional Doppler, causing a bulk shift of the image in the Doppler domain. However, it brings serious range migration, which is expected to be corrected in the conventional range alignment step. And, the presence of the high-order terms introduces both range migration and phase errors. Based on the polynomial translation model, we rewrite the signal in (10) as follows.

$$
\begin{aligned}
s(m, n)= & \widetilde{s}(m, n) \cdot \exp \left[-j 4 \pi \frac{R(n)}{c} \cdot\left(m \cdot \Delta f_{r}+f_{c}\right)\right]+\varepsilon_{F}(m, n) \\
= & \widetilde{s}(m, n) \cdot \exp \left[-j 4 \pi \frac{\sum_{k=1}^{K} \alpha_{k}(n \cdot \Delta t)^{k}}{c} \cdot\left(m \cdot \Delta f_{r}+f_{c}\right)\right] \\
& +\varepsilon_{F}(m, n)
\end{aligned}
$$

For simplicity and clarity, we define the polynomial coefficient vector as $\alpha=\left[\begin{array}{lll}\alpha_{1}, & \cdots, & \alpha_{K}\end{array}\right]_{1 \times K}$, and give the complex image after error correction by $\widetilde{\alpha}=$ $\left[\begin{array}{lll}\widetilde{\alpha}_{1} & \cdots & \widetilde{\alpha}_{K}\end{array}\right]_{1 \times K}$ as follows.

$$
\begin{aligned}
g(k, h ; \widetilde{\alpha})= & \frac{1}{N M} \sum_{n=0}^{N-1} \exp \left(j 2 \pi \frac{h n}{N}\right) \cdot \sum_{m=0}^{M-1} \exp \left(j 2 \pi \frac{k m}{M}\right) \\
& \cdot s(m, n) \cdot \exp \left[j 4 \pi \frac{\sum_{k=1}^{K} \widetilde{\alpha}_{k}(n \cdot \Delta t)^{k}}{c} \cdot\left(m \cdot \Delta f_{r}+f_{c}\right)\right]
\end{aligned}
$$

Therefore, the entropy of image is defined as function of $\widetilde{a}$, which is given by

$$
E_{g}(\widetilde{\alpha})=-\frac{1}{S_{g}} \sum_{k=0}^{M-1} \sum_{h=0}^{N-1}|g(k, h ; \widetilde{\alpha})|^{2} \ln |g(k, h ; \widetilde{\alpha})|^{2}+\ln S_{g}
$$

where the image intensity is rewritten by

$$
S_{g}=\sum_{k=0}^{M-1} \sum_{h=0}^{N-1}|g(k, h ; \widetilde{\alpha})|^{2}
$$

We note that as the motion correction in (24) is performed on the phase of $s(m, n)$. And, the signal energy holds constant according to the Parseval theorem [30]. As a result, $S_{g}$ is independent of $\widetilde{a}$. The estimate of $\alpha$ is obtained by minimizing the image entropy, expressed as follows.

$$
\langle\hat{\alpha}\rangle=\underset{\widetilde{\alpha}}{\arg \min } E_{g}(\widetilde{\alpha})
$$

To solve this optimization, many standard algorithms are available, such as gradient-based algorithms, genetic algorithms, and gold section search. Due to the random characteristics of noise, it contributes little to the variance of entropy during the motion estimation $[19,22]$. And, some dominant scatters will exceed the noise in amplitude distinctively due to energy accumulation when optimal motion correction is achieved, enhancing the sharpness of image significantly. Therefore, minimizing the entropy of 2D image for error correction is reasonable. Due to the strong noise interference, the cost function is usually not convex along with a lot of local minimums. Straightforwardly, utilizing the standard optimization algorithms encounters high risk that the iteration is trapped into a local minimum that far away from the true solution. In this article, we propose a coordinate descent algorithm implemented by quasiNewton algorithm. The coordinate descent algorithm sequentially minimizes the objective function with respect to a single parameter while holding the remaining parameters constant. By this, coordinate decent algorithms have monotone convergence in the objective function and trend to have the ability of achieving the global optimization [19].

Let $\widetilde{\alpha}^{(l)}=\left[\widetilde{\alpha}_{1}^{(l)}, \ldots, \widetilde{\alpha}_{K}^{(l)}\right]_{1 \times K}$ denote the estimate of the $l$ th iteration, and $\widetilde{\alpha}^{(l, k)}$ be the estimate of the phase parameters at the $l$ th iteration where the first $k-1$ polynomial coefficients have already been updated.

$$
\widetilde{\alpha}^{(l, k)}=\left[\widetilde{\alpha}_{1}^{(l+1)}, \ldots, \widetilde{\alpha}_{k-1}^{(l+1)}, \widetilde{\alpha}_{k}^{(l)}, \widetilde{\alpha}_{k+1}^{(l)}, \ldots, \widetilde{\alpha}_{K}^{(l)}\right]_{1 \times K}
$$

where an iteration is defined as a complete cycle through all $K$ coefficients. The task in hand now is to 
update the $k$ th coefficient. Assuming that the update is denoted by a variable $\Delta \widetilde{\alpha}_{k}$, we have the corrected image as follows.

$$
\begin{aligned}
g\left(k, h ; \widetilde{\alpha}^{(l, k)} ; \Delta \widetilde{\alpha}_{k}\right)= & \frac{1}{N M} \sum_{n=0}^{N-1} \exp \left(j 2 \pi \frac{h n}{N}\right) \cdot \sum_{m=0}^{M-1} \exp \\
& \times\left(j 2 \pi \frac{k m}{M}\right) \cdot s_{c}\left(m, n ; \widetilde{\alpha}^{(l, k)}\right) \\
& \cdot \exp \left[j 4 \pi \frac{\Delta \widetilde{\alpha}_{k} \cdot(n \cdot \Delta t)^{k}}{c} \cdot\left(m \cdot \Delta f_{r}+f_{c}\right)\right]
\end{aligned}
$$

where

$$
\begin{aligned}
s_{c}\left(m, n ; \widetilde{\alpha}^{(l, k)}\right)= & s(m, n) \\
& \cdot \exp \left[j 4 \pi \frac{\sum_{u=1}^{\tilde{K}} \widetilde{\alpha}^{(l, k)}(u) \cdot(n \cdot \Delta t)^{u}}{c} \cdot\left(m \cdot \Delta f_{r}+f_{c}\right)\right]
\end{aligned}
$$

And the entropy function is rewritten by

$$
\begin{aligned}
E_{g}\left(\widetilde{\alpha}^{(l, k)} ; \Delta \widetilde{\alpha}_{k}\right)= & -\frac{1}{S_{g}} \sum_{k=0}^{M-1} \sum_{h=0}^{N-1}\left|g\left(k, h ; \widetilde{\alpha}^{(l, k)} ; \Delta \widetilde{\alpha}_{k}\right)\right|^{2} \\
& \times \ln \left|g\left(k, h ; \widetilde{\alpha}^{(l, k)} ; \Delta \widetilde{\alpha}_{k}\right)\right|^{2}+\ln S_{g}
\end{aligned}
$$

The image intensity is

$$
S_{g}=\sum_{k=0}^{M-1} \sum_{h=0}^{N-1}\left|g\left(k, h ; \widetilde{\alpha}^{(l, k)} ; \Delta \widetilde{\alpha}_{k}\right)\right|^{2}
$$

The estimates of update for $\widetilde{\alpha}_{k}$ in the $l$ th iteration is given by minimizing the image entropy, expressed as follows

$$
\begin{aligned}
\left\langle\Delta \widetilde{\alpha}_{k}^{(l+1)}\right\rangle & =\underset{\widetilde{\Delta \alpha_{k}}}{\arg \min } E_{g}\left(\widetilde{\alpha}^{(l, k)} ; \Delta \widetilde{\alpha}_{k}\right) \text { and } \widetilde{\alpha}_{k}^{(l+1)} \\
& =\widetilde{\alpha}_{k}^{(l)}+\Delta \widetilde{\alpha}_{k}^{(l+1)}
\end{aligned}
$$

To solve this one-dimensional optimization, we utilize the quasi-Newton algorithm, where $\Delta \widetilde{\alpha}_{k}$ minimizing $E_{g}\left(\widetilde{\alpha}^{(l, k)} ; \Delta \widetilde{\alpha}_{k}\right) \quad$ satisfies that the derivatives of $E_{g}\left(\widetilde{\alpha}^{(l, k)} ; \Delta \widetilde{\alpha}_{k}\right)$ with respect to $\Delta \widetilde{\alpha}_{k}$ equals zero. The derivative of $\partial E_{g}\left(\widetilde{\alpha}^{(l, k)} ; \Delta \widetilde{\alpha}_{k}\right)$ with respect to $\Delta \widetilde{\alpha}_{k}$ is given by

$$
\begin{aligned}
& \nabla E_{g}\left(\widetilde{\alpha}^{(l, k)} ; \Delta \widetilde{\alpha}_{k}\right)=\frac{\partial E_{g}\left(\widetilde{\alpha}^{(l, k)} ; \Delta \widetilde{\alpha}_{k}\right)}{\partial \Delta \widetilde{\alpha}_{k}} \\
& =-\frac{1}{S_{g}} \sum_{k=0}^{M-1 N-1}\left[1+\ln \left|g\left(k, h ; \widetilde{\alpha}^{(l, k)} ; \Delta \widetilde{\alpha}_{k}\right)\right|^{2}\right] \\
& \frac{\partial\left|g\left(k, h ; \widetilde{\alpha}^{(l, k)} ; \Delta \widetilde{\alpha}_{k}\right)\right|^{2}}{\partial \Delta \alpha_{k}}
\end{aligned}
$$

Since $\quad\left|g\left(k, h ; \widetilde{\alpha}^{(l, k)} ; \Delta \widetilde{\alpha}_{k}\right)\right|^{2}=g\left(k, h ; \widetilde{\alpha}^{(l, k)} ; \Delta \widetilde{\alpha}_{k}\right) \cdot g^{*}$ $\left(k, h ; \widetilde{\alpha}^{(l, k)} ; \Delta \widetilde{\alpha}_{k}\right)$, we have

$$
\begin{aligned}
\frac{\partial\left|g\left(k, h ; \widetilde{\alpha}^{(l, k)} ; \Delta \widetilde{\alpha}_{k}\right)\right|^{2}}{\partial \Delta \widetilde{\alpha}_{k}}= & g^{*}\left(k, h ; \widetilde{\alpha}^{(l, k)} ; \Delta \widetilde{\alpha}_{k}\right) \frac{\partial g\left(k, h ; \widetilde{\alpha}^{(l, k)} ; \Delta \widetilde{\alpha}_{k}\right)}{\partial \Delta \widetilde{\alpha}_{k}} \\
& +g\left(k, h ; \widetilde{\alpha}^{(l, k)} ; \Delta \widetilde{\alpha}_{k}\right) \frac{\partial g^{*}\left(k, h ; \widetilde{\alpha}^{(l, k)} ; \Delta \widetilde{\alpha}_{k}\right)}{\partial \Delta \widetilde{\alpha}_{k}} \\
= & 2 \cdot \operatorname{Re}\left[g^{*}\left(k, h ; \widetilde{\alpha}^{(l, k)} ; \Delta \widetilde{\alpha}_{k}\right) \frac{\partial g\left(k, h ; \widetilde{\alpha}^{(l, k)} ; \Delta \widetilde{\alpha}_{k}\right)}{\partial \Delta \widetilde{\alpha}_{k}}\right]
\end{aligned}
$$

where

$$
\frac{\partial g\left(k, h ; \widetilde{\alpha}^{(l, k)} ; \Delta \widetilde{\alpha}_{k}\right)}{\partial \Delta \widetilde{\alpha}_{k}}=\frac{j 4 \pi}{N} \cdot \sum_{n=0}^{N-1} \exp \left(j 2 \pi \frac{h n}{N}\right) \cdot y\left(k, n ; \widetilde{\alpha}^{(l, k)} ; \Delta \widetilde{\alpha}_{k}\right),
$$

and

$$
y\left(k, n ; \widetilde{\alpha}^{(l, k)} ; \Delta \widetilde{\alpha}_{k}\right)=\frac{1}{M} \sum_{m=0}^{M-1} \exp \left(j 2 \pi \frac{k m}{M}\right) \cdot s_{c}\left(m, n ; \widetilde{\alpha}^{(l, k)}\right)
$$

$$
\cdot \exp \left[j 4 \pi \frac{\Delta \widetilde{\alpha}_{k} \cdot(n \cdot \Delta t)^{k}}{c} \cdot\left(m \cdot \Delta f_{r}+f_{c}\right)\right]
$$

$$
\cdot(n \cdot \Delta t)^{k} \cdot\left(\frac{m \cdot \Delta f_{r}+f_{c}}{c}\right),
$$

In order to implement the quasi-Newton algorithm, we also need the second-order derivative of $E_{g}\left(\widetilde{\alpha}^{(l, k)} ; \Delta \widetilde{\alpha}_{k}\right)$ with respect to $\Delta \widetilde{\alpha}_{k}$, which is given by 


$$
\begin{aligned}
& \nabla^{2} E_{g}\left(\widetilde{\alpha}^{(l, k)} ; \Delta \widetilde{\alpha}_{k}\right)=\frac{\partial^{2} E_{g}\left(t_{e} ; \phi\right)}{\partial \Delta \widetilde{\alpha}_{k} \partial \Delta \widetilde{\alpha}_{k}} \\
& =-\frac{1}{S_{g}} \sum_{k=0}^{M-1 N-1} \sum_{h=0}\left[\begin{array}{l}
\left(1+\ln \left|g\left(k, h ; \widetilde{\alpha}^{l, k)} ; \Delta \widetilde{\alpha}_{k}\right)\right|^{2}\right) \cdot \frac{\partial^{2}\left|g\left(k, h ; \widetilde{\alpha}^{(l, k)} ; \Delta \widetilde{\alpha}_{k}\right)\right|^{2}}{\partial \Delta \widetilde{\alpha}_{k} \partial \Delta \widetilde{\alpha}_{k}} \\
+\frac{1}{\left|g\left(k, h ; \widetilde{\alpha}^{(l, k)} ; \Delta \widetilde{\alpha}_{k}\right)\right|^{2}} \cdot\left(\frac{\partial\left|g\left(k, h ; \widetilde{\alpha}^{(l, k)} ; \Delta \widetilde{\alpha}_{k}\right)\right|^{2}}{\partial \Delta \widetilde{\alpha}_{k}}\right)^{2}
\end{array}\right] \\
& =a\left(\widetilde{\alpha}^{(l, k)} ; \Delta \widetilde{\alpha}_{k}\right)+b\left(\widetilde{\alpha}^{(l, k)} ; \Delta \widetilde{\alpha}_{k}\right)
\end{aligned}
$$

In Appendix, explicit expressions of $a\left(\widetilde{\alpha}^{(l, k)} ; \Delta \widetilde{\alpha}_{k}\right)$ and $b\left(\widetilde{\alpha}^{(l, k)} ; \Delta \widetilde{\alpha}_{k}\right)$ are deduced. Then, $\Delta \widetilde{\alpha}_{k}$ is estimated iteratively, which is defined as the inner iteration of the joint motion correction. It should be emphasized that, in the quasi-Newton algorithm, all IDFT calculation can be implemented by fast Fourier transform, yielding significant efficiency improvement. The $p$ th update is given by

$$
\begin{aligned}
& \Delta \widetilde{\alpha}_{k}^{(p+1)}=\Delta \widetilde{\alpha}_{k}^{(p)}+d^{(p+1)} \\
& d^{(p)}=-\left[\nabla^{2} E_{g}\left(\widetilde{\alpha}^{(l, k)} ; \Delta \widetilde{\alpha}_{k}^{(p)}\right)\right]^{-1} \cdot \nabla E_{g}\left(\widetilde{\alpha}^{(l, k)} ; \Delta \widetilde{\alpha}_{k}^{(p)}\right) \\
& 1 \leq p \leq P
\end{aligned}
$$

where $P$ denotes the total number of iterations. In updating of the $k$ th coefficients in the $l$ th outer iteration, the corrected image yields

$$
\begin{aligned}
& g\left(k, h ; \widetilde{\alpha}^{(l, k)} ; \Delta \widetilde{\alpha}_{k}^{(p+1)}\right) \\
& =\frac{1}{N M} \sum_{n=0}^{N-1} \exp \left(j 2 \pi \frac{h n}{N}\right) \cdot \sum_{m=0}^{M-1} \exp \left(j 2 \pi \frac{k m}{M}\right) \cdot s_{c}\left(m, n ; \widetilde{\alpha}^{(l, k)}\right) \\
& \quad \cdot \exp \left[j 4 \pi \frac{\Delta \widetilde{\alpha}_{k}^{(p+1)} \cdot(n \cdot \Delta t)^{k}}{c} \cdot\left(m \cdot \Delta f_{r}+f_{c}\right)\right]
\end{aligned}
$$

We increase $p$ and go back to the update in (39) until $E_{g}\left(\widetilde{\alpha}^{(l, k)} ; \Delta \widetilde{\alpha}_{k}^{(p)}\right)-E_{g}\left(\widetilde{\alpha}^{(l, k)} ; \Delta \widetilde{\alpha}_{k}^{(p+1)}\right) \leq \rho$, where $\rho$ is a parameter small enough for the pre-determined threshold or $p$ reaches a pre-determined maximum iteration number $P$. For computational efficiency, fast Fourier transform should be used in the implementation of the calculation above.

With the polynomial coefficients being updated sequentially, the image entropy decreases stage-by-stage. And, with the iteration number increases, convergence can be obtained. The convergence of the outer loop can be determined by $E_{g}\left(\widetilde{\alpha}^{(l)}\right)-E_{g}\left(\widetilde{\alpha}^{(l+1)}\right) \leq \beta$, where $\beta$ is the pre-set tolerance or $l$ reaches a pre-set maximum number $L$. For clarity, we present the flowchart of the coordinate descent motion correction in Figure 2, where $\widetilde{\alpha} i$ is the initialization of $\widetilde{\alpha}$, which will be discussed in the following section. From Figure 2, we note the polynomial coefficients are estimated iteratively by using an outer loop and an inner loop. The outer loop corresponds to the update of the polynomial coefficient vector and the inner loop is implemented by the quasiNewton algorithm to update a single component in the vector. In general, for this inner loop, we usually need only several iterations for a single component $\widetilde{\alpha}^{(l, k)}$. The polynomial coefficients are updated by the quasiNewton algorithm sequentially, and for the update of each coefficient, several iterations are needed. As a result, we need to perform the translational motion correction and image processing several times for every component, and high computational load may be involved. However, the monotone and fast convergence is usually achieved by the coordinate descent algorithm even under low SNR, and as we apply the quasi-Newton algorithm in the inner loop, its fast convergence also promotes the algorithm in efficiency. It should be emphasized that we usually have no additional knowledge of the translational motion in reality, and the polynomial order $K$ should be selected manually or adaptively. A pre-determined order should be high enough to model the real motion, which causes high computational complexity. In the following section, we will develop an adaptive selection approach.

\subsection{Coarse motion estimation for initialization}

A significant aspect on the computational efficiency is the initialization of $\widetilde{\alpha}$. Like any optimization algorithm, it is beneficial to start as close to the correct solution as possible to improve the convergence. However, this information is usually unavailable in reality. In this sense, before performing the coordinate descent algorithm, we can estimate a coarse candidate of $\widetilde{\alpha}$ at a small computational price. Herein, we propose an efficient approach to achieve the coarse estimate $\widetilde{\alpha} i=\left[\begin{array}{lll}\widetilde{\alpha} i_{1} & \cdots & \widetilde{\alpha} i_{\bar{K}}\end{array}\right]_{1 \times \bar{K}}$, where $\bar{K}$ is set large enough to model the real translational motion. Similar to the coordinate descent algorithm, in the estimation of $\widetilde{\alpha} i_{k}$, all rest components are held constant. The coarse motion estimation is implemented by means of an exhaustive linear search, over the variable $\widetilde{\alpha} i_{k}$, within a pre-defined interval $\left[\alpha i_{k}^{\mathrm{min}}\right.$, $\left.\alpha i_{k}^{\max }\right]$. Similar to the coarse acceleration estimation in [14], the choice the search step is heuristic as a large search step causes precision degradation and a small one involves large computational load. In general, the image entropy function with respect to $\widetilde{\alpha} i_{k}$ is usually smooth, and we can use only a number of discrete samples to recover the entire entropy function by interpolation. In 


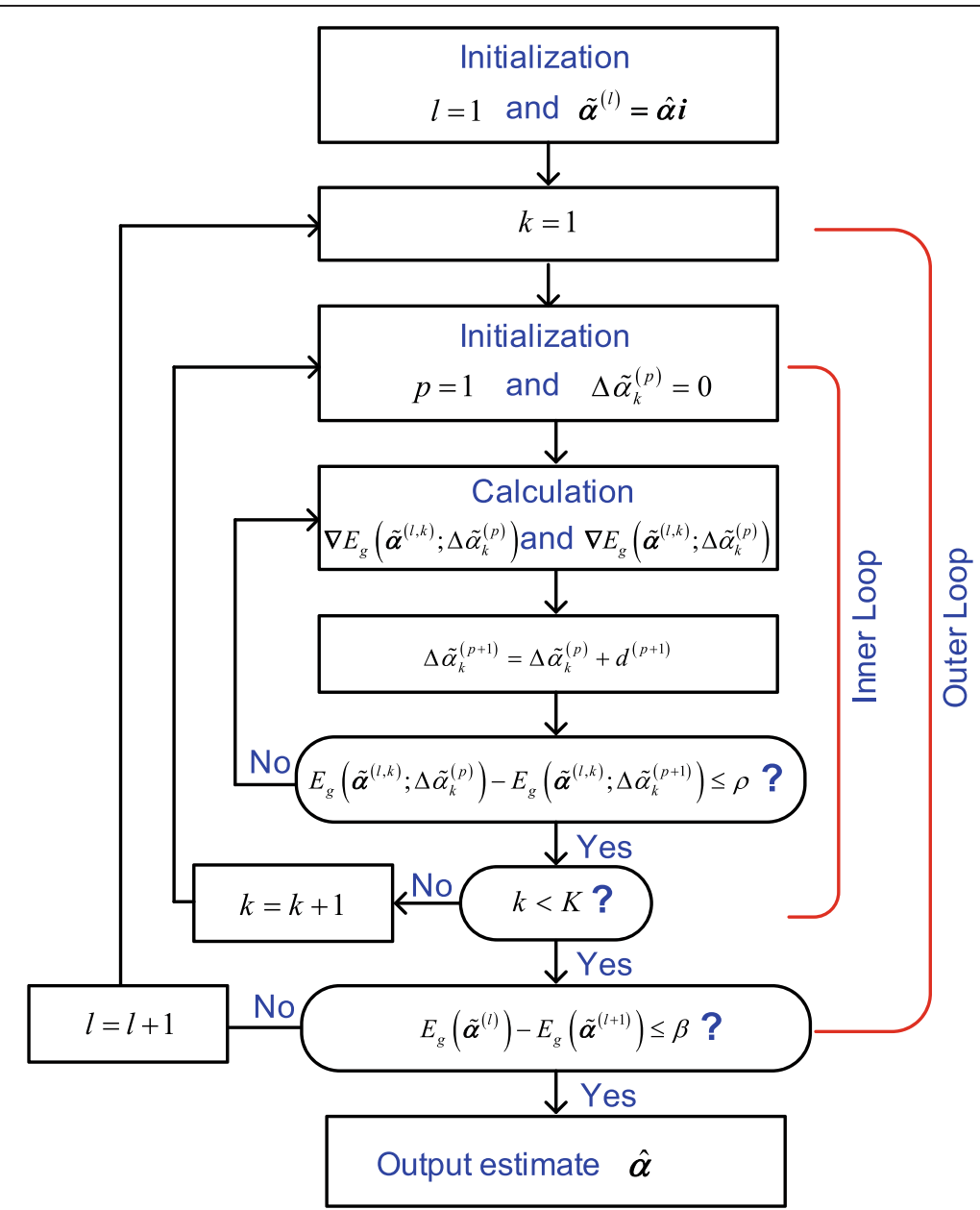

Figure 2 Flowchart of the coordinate descent algorithm.

this sense, the search interval should be large enough to capture the true value, but the search step between two discrete samples could be set large for high efficiency. The coarse estimate of $\widetilde{\alpha} i_{k}$ is determined by finding the minimum on the interpolated entropy function. It should be emphasized that the smoothness of the image entropy function is elementary to the interpolation. And in reality, the function would fluctuate seriously under strong noise, presenting several local minimums. In this situation, the search step should be set narrow enough to avoid missing the global minimum. For clarity, we give an example of estimation of the velocity in Figure 3. The velocity and acceleration of the target are $20 \mathrm{~m} / \mathrm{s}$ and $8.5 \mathrm{~m} / \mathrm{s}^{2}$, and $\mathrm{SNR}=-7 \mathrm{~dB}$. The curve is interpolated from only 10 samples, which presents three local minimums, and the estimate of 18.3 is close to the ideal velocity. However, as the SNR further decreases, the entropy function curve fluctuates more greatly, leading to large bias of coarse motion estimation by the interpolation. For performance enhancement, the coarse motion estimation is implemented in an iterative

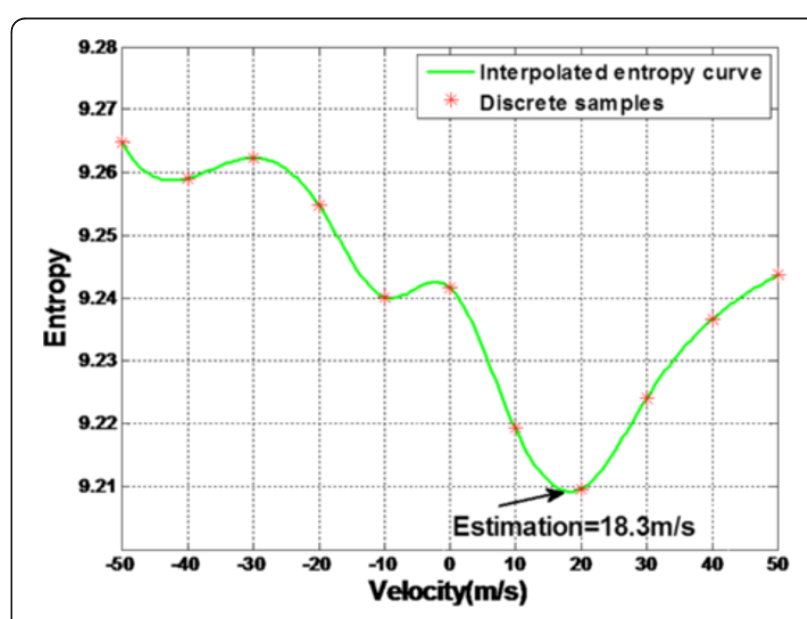

Figure 3 Coarse estimation of ãi. 
manner, as shown in Figure 4. In general, the method is capable of providing the useful coarse translational motion compensation even if strong noise presents, and the residual motion can be estimated by the coordinate descent optimization. In terms of further accelerating the minimum entropy approach, parallelized implementation of the linear search within the iteration can be applied as the calculation for each sample is independent with of each other. Moreover, the search procedure in [31] can be also applied. By decreasing both the search interval and step with the increase of iteration number, high accuracy and efficiency can be achieved. Another important aspect should be taken into account, which is the order selection of the polynomial model in (28). Herein, the order estimation is embedded into the coarse motion estimation. In general, for a rigid target, the low-order motion terms take up the majority of the translational motion, while the higher-order motion is nominal. The order of $\widetilde{\alpha}$ is determined adaptively, and it is assumed to be accurate enough when the estimates of two consecutive $\tilde{\alpha} i_{k}$ are smaller than a pre-determined threshold. The threshold is so small that the motion under it does not change the image entropy. In general, a threshold of $1 \mathrm{e}-3$ is usually a good choice in reality.

In [14], a novel joint correction approach to motion estimation is developed, which models the translational motion as a two-order polynomial function determined by the radial velocity and acceleration. And, a novel coarse estimation method is also developed, where the radial velocity and acceleration are estimated by Radon Transform (RT) and IC, respectively. Utilizing RT for velocity estimation is very similar to the Hough-based range alignment approach [5]. Assuming the linear migration of the range profiles is mainly induced by the radial velocity, the velocity can be determined by the slope of range profiles estimated by RT [14]. Subsequently, maximum IC optimization is applied to obtain the radial acceleration. RT is capable of providing precise estimation of the radial velocity, as it accumulates energy of signal in a non-coherent way. However, its precision degrades with the increase of the noise. And, strong noise can submerge the range profiles causing that the SNR gain from non-coherent accumulation would not be great enough to achieve accurate velocity estimation.

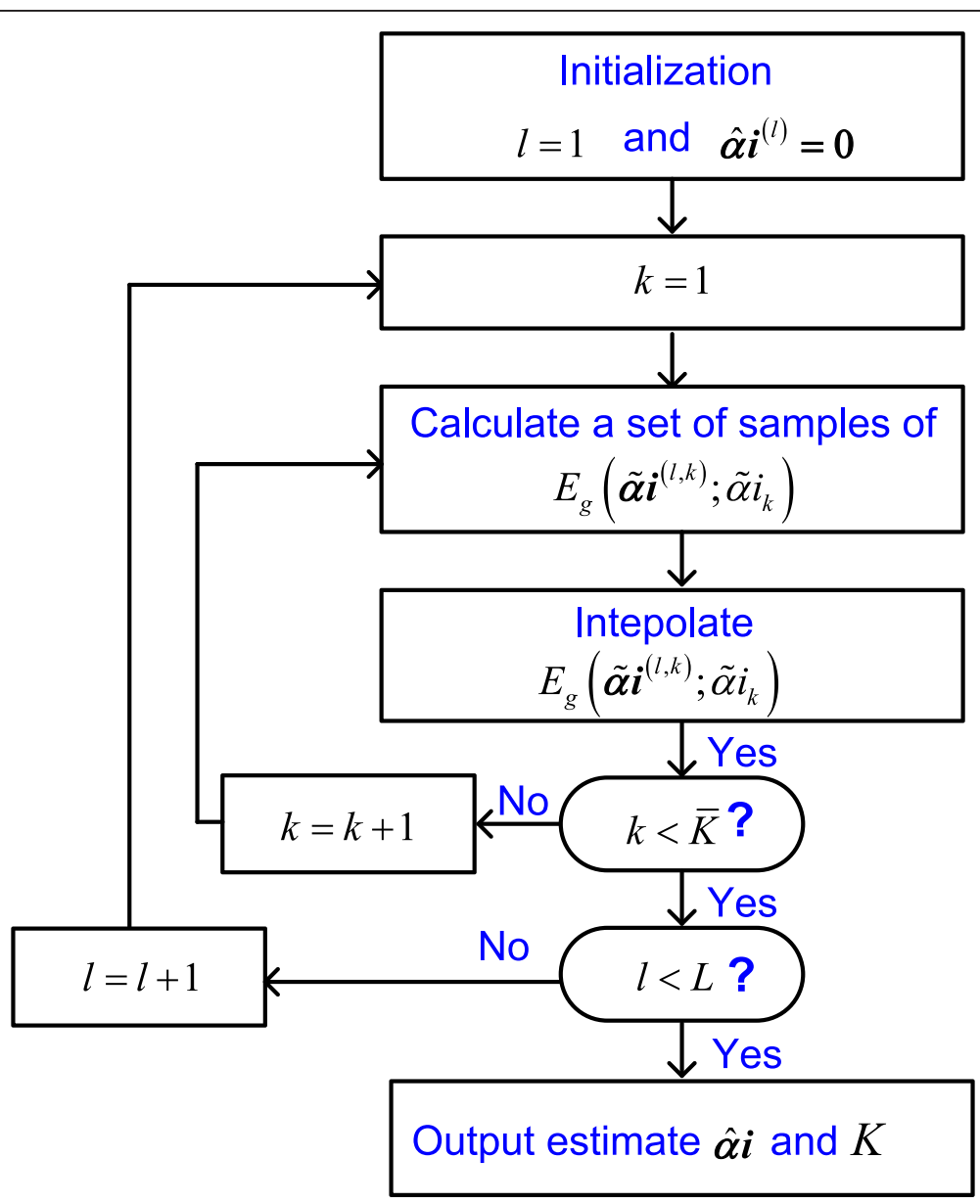

Figure 4 Flowchart of the coarse motion estimate. 
Subsequently, the acceleration estimation with contrast maximization fails. Obviously, the proposed coarse motion estimation with minimum entropy achieves the high-SNR gain from the coherent accumulation since it evaluates the entropy of the final ISAR image. On the other hand, the minimum entropy approach iteratively estimates the motion coefficients in sequence via the linear search, which is deemed to involve relatively large computational load. In this sense, the proposed coarse estimation should be workable under lower SNR than the RT and IC method at a price of increased computational load. To present the superiority and shortcoming of the proposed approach for coarse motion estimation under low-SNR conditions, herein we use synthetic data for comparison. The synthetic data are generated by adding different Gaussian white noise into the real Yak42 dataset (the dataset will be depicted in the following section), yielding $\mathrm{SNR}=-7 \mathrm{~dB}$ and $-10 \mathrm{~dB}$. The velocity and acceleration of the target are set as $20 \mathrm{~m} / \mathrm{s}$ and $8.5 \mathrm{~m} / \mathrm{s}^{2}$, respectively. Then, both of the coarse motion estimation approaches are utilized to obtain the radial motion coefficients. In the case of SNR $=-7 \mathrm{~dB}$, both of them work well, providing precise estimation of both velocity and acceleration. In Figure 5a, we plot the ideal radial range together with the estimated ones. From Figure $5 \mathrm{a}$, one can note that the estimations are very close to the idea radial range curves. And, the RT and IC-based method performs only slightly worse than the minimum entropy method. However, as shown in Figure 5b, when SNR decreases down to $-10 \mathrm{~dB}$, the RT and IC-based coarse estimation fails, while the performance of the minimum entropy method maintains. It should be noted that about five iterations are needed to ensure the convergence of the minimum entropy-based initialization, and its computational load is over five times that of the RT and ICbased method. Both of them are coded in Matlab language with the same computing environment. In experiments, we find that the RT and IC-based coarse estimation fails when SNR decreases below $-8 \mathrm{~dB}$, while the minimum entropy-based coarse estimation is still workable until SNR decreases down to $-12 \mathrm{~dB}$ due to high-SNR gain from the coherent accumulation. For both maximum contrast and minimum entropy optimization, the initialization step is so essential that only an effective initialization ensures precise estimation by using gradient-based optimization algorithms. And, the proposed coordinate descent algorithm and the one utilized in [14] are not exceptions. In this sense, the convergence of the optimization algorithms to the global optimization depends on the coarse estimation significantly. Because both entropy and contrast functions show good convexity and smoothness only near to the global extremum and strong multimodal behavior presents in the position far from it. Moreover, the fluctuation phenomenon of cost function becomes furthermore dis- tinctive when noise increases. In low-SNR situations, optimal coarse estimation is capable of accelerating optimization algorithms to reach the global optimization. On contrary, a bad initialization would lead the algorithms to be trapped within a local optimal point far away from the global optimization. To further demonstrate this, we provide the image results under different SNRs by using the maximum IC method in [14] and the proposed minimum entropy method. In the maximum IC method, after the RT and IC initialization, maximum IC optimization is solved by the Nelder and Mead's method [14]. And, in our scheme, the coordinate descent algorithm is applied after the minimum entropy coarse compensation. In Figure 5c, we present the ideal image and images obtained by the two methods under SNR $=-7 \mathrm{~dB}$. They are arrayed from left to right in sequence. And, Figure $5 \mathrm{~d}$ shows the results with $\mathrm{SNR}=-10 \mathrm{~dB}$. In the case of SNR $=-7 \mathrm{~dB}$, since both the initialization algorithms perform well, the starting guesses are close to the global optimization, and we also find that it is very flexible and simple to set parameters (such as reflection and expansion coefficients in Nelder and Mead's method) in both maximum IC and minimum entropy optimizations. And, both of them achieve well-focused images. However, in the case of SNR $=-10 \mathrm{~dB}$, as the RT and IC initialization fails to provide an effective initialization for the Nelder and Mead's approach, it consequently fails to achieving the true motion coefficients, leading to blurred image, while thanks to the 2D coherent integrant SNR gain, minimum entropy initialization provides precise coarse coefficients, and the coordinate decent algorithm reaches the optimal convergence with only about three iterations. And, the resulting RD image after correction is very close to the ideal one as shown in Figure 5d. From the comparison, we can conclude that, in low-SNR circumstances, initialization is essentially significant for metric optimization-based motion compensation. Since strong noise usually induces fluctuation in the cost function and it is only smooth near to the global extremum. Only effective initialization can support the following fine estimation by deterministic optimization algorithms to get the global optimization. And we should also note, this dependence of initialization may be reduced by heuristic optimization algorithms, such as genetic algorithm [32] and particle swarm optimization [33] at a price of increased computational load.

\section{Performance analysis}

In this section, real datasets are used to evaluate the performance of the joint range alignment and phase adjustment for ISAR imaging. Contrast, entropy and peak value of the focused RD images are used as the evaluation criterion of the image quality. First, the Yak-42 data are utilized to analyze the performance of the proposed method. High-order polynomial motion and different noise are 


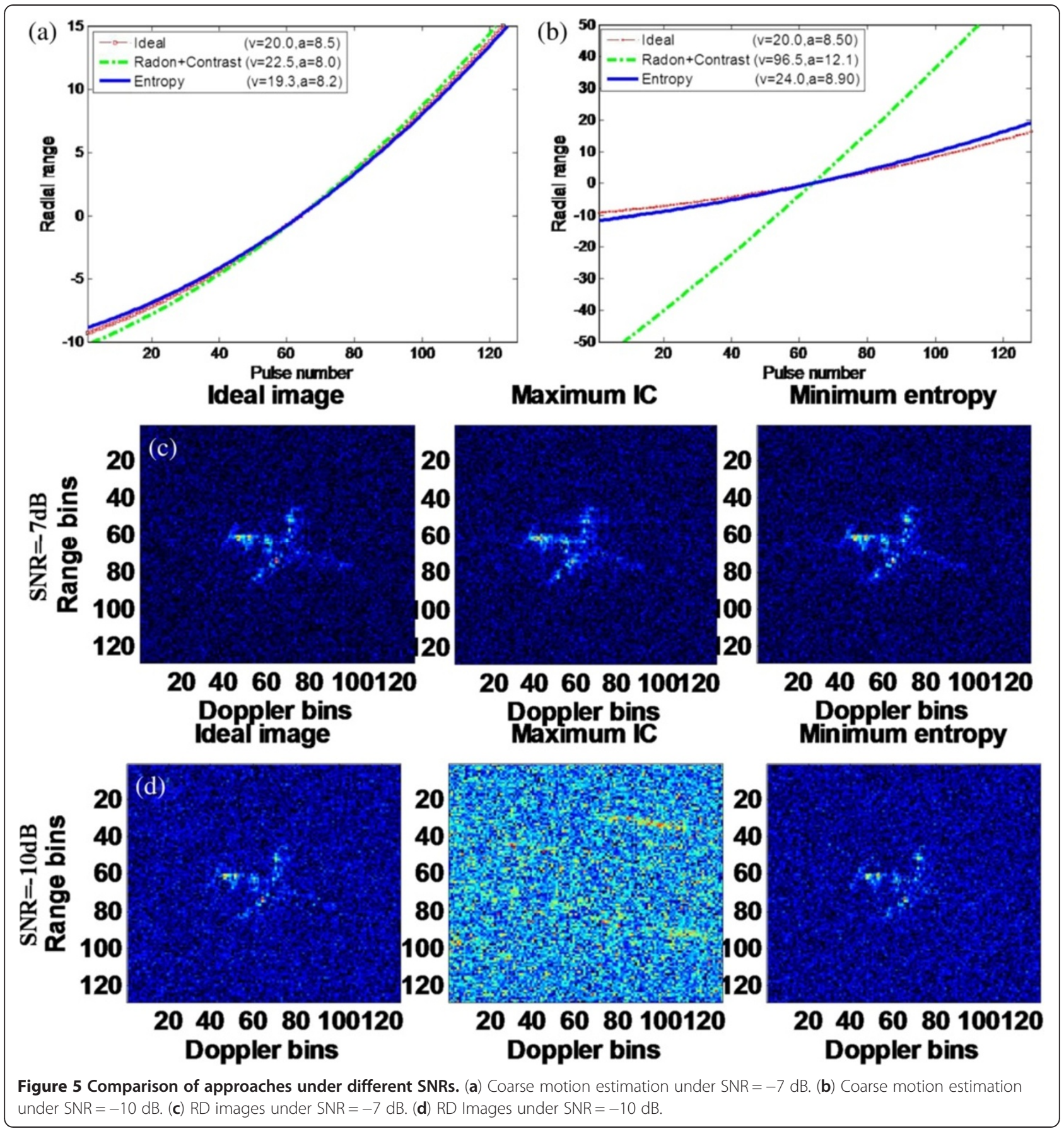

added into the data, and the motion compensation is performed by both the conventional minimum entropybased methods and the proposal. Comparisons are also provided to illuminate the improvement of our method. A ship data with strong sea clutter is also used to confirm the effectiveness of our method. All results illuminate that the enhanced quality is achieved by the joint correction.

The dataset of the Yak-42 airplane is recorded by a Cband $(5.52 \mathrm{GHz})$ ISAR experimental system. The system transmits $400 \mathrm{MHz}$ linear modulated chirp signal with
$25.6 \mu$ s pulse duration, providing a range resolution of $0.375 \mathrm{~m}$. In the dataset, the pulse repetition frequency is $100 \mathrm{~Hz}$, i.e., 128 pulses within dwell time [-0.64, 0.64] (s) are used in the following experiment. Conventional motion compensation, including MERA [9] and MEPA [19], is performed to remove the translational motion. Due to high SNR (up to $22 \mathrm{~dB}$ ) of the raw data, both of them perform well. The aligned profiles are shown in Figure 6a. And RD image is shown in Figure 6b for evaluating the experimental results. 

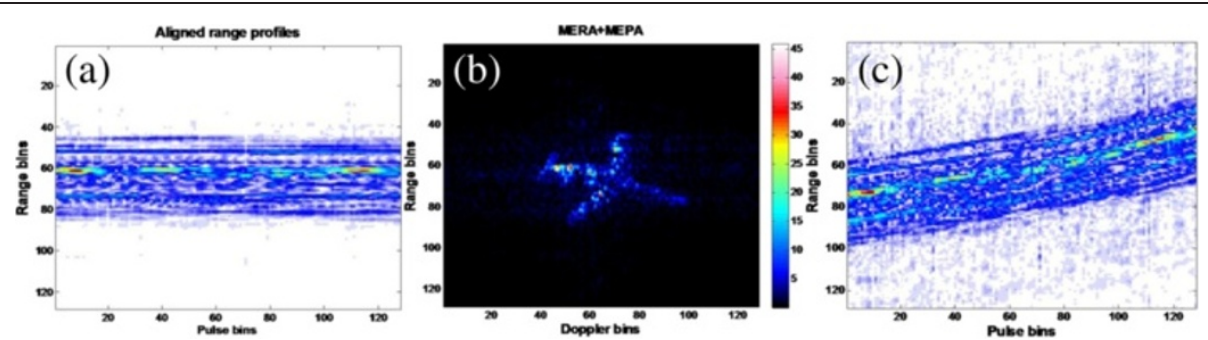

Figure 6 Experimental dataset. (a) Aligned range profiles. (b) RD image via MERA + MEPA. (c) Range profiles with motion error.

Herein, we use the Yak-42 data to generate synthetic data with different SNRs. The high-order motion error with $\alpha=$ $[1-3,5,10,30]$ is added into the corrected data causing misalignment and phase error. The misaligned profiles are shown in Figure 6c. Complex white Gaussian noise is added to generate different SNRs (from 5 to $-20 \mathrm{~dB}$ ). For comparison, conventional motion correction is implemented by the MERA and MEPA, where we use MEPA with simultaneous update scheme [18] due to its computational efficiency and robustness. As discussed before, MERA is usually more sensitive to strong noise than MEPA. We perform MEPA to the aligned HRRPs as shown in Figure 6a under different SNRs and then generate RD images. Thanks to the robustness of MEPA, the images are wellfocused and they can be regarded as the standards for comparison. These standard images are shown in the left column of Figure 7 corresponding to SNR equivalent to 5, $0,-5$, and $-10 \mathrm{~dB}$ by CPU times $2.00,3.14,6.71$, and 10.04 s, respectively. RD images obtained by MERA and MEPA are shown in the second column of Figure 7 corres-

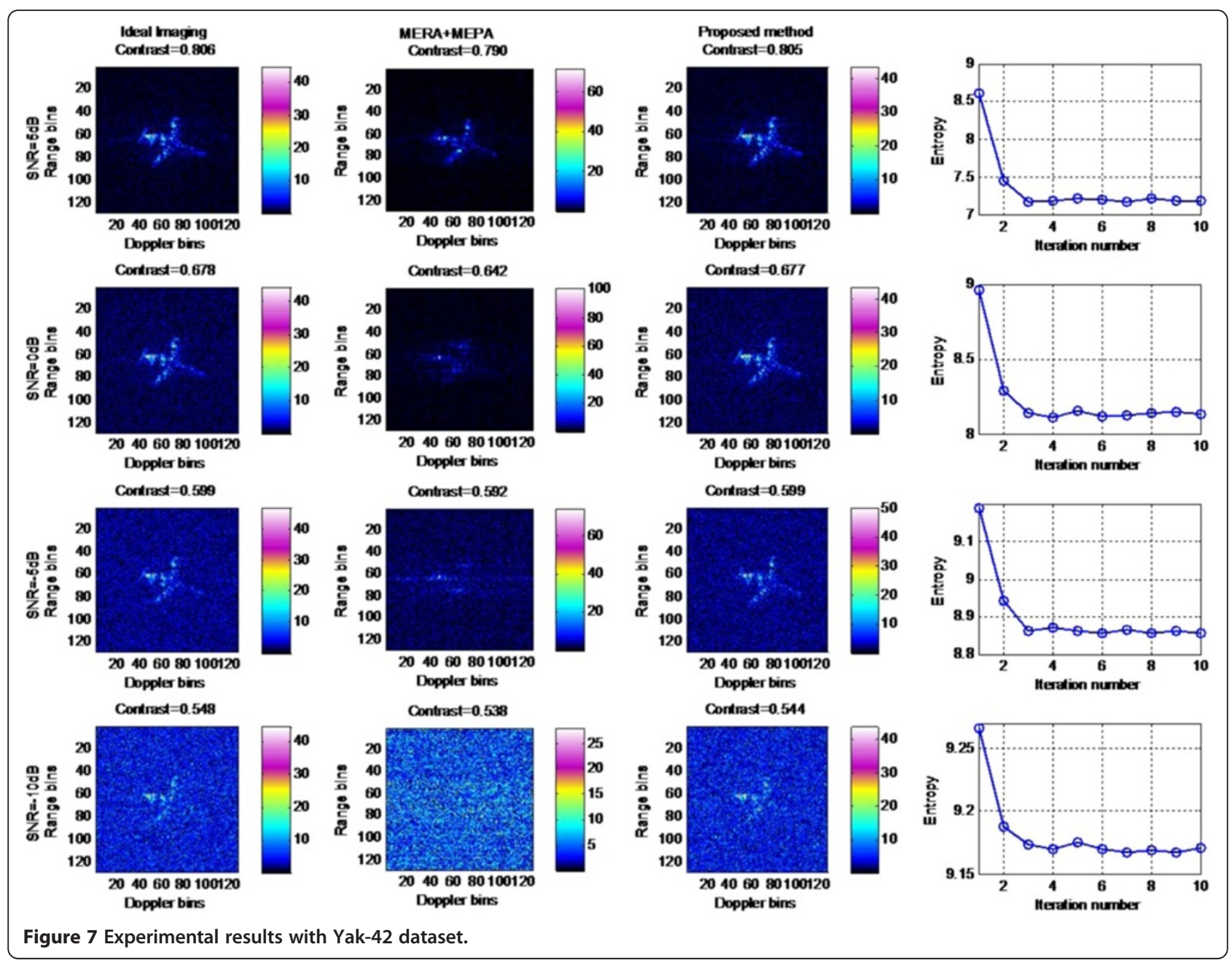




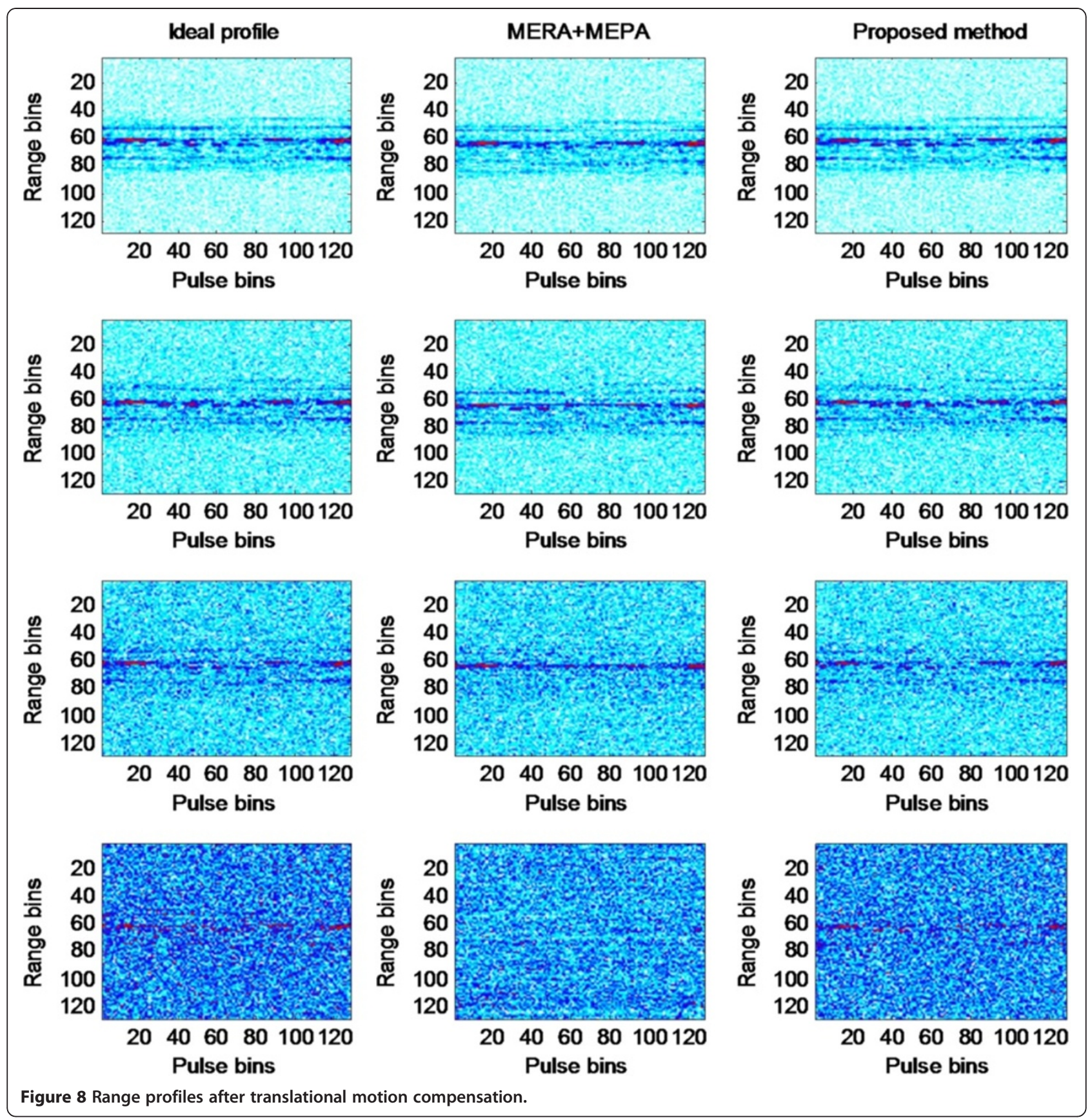

ponding to different SNRs. The images from the joint correction are given in the third column of Figure 7 . And the CUP time for the joint correction under different SNRs is $5.65,8.09,10.12$, and $13.20 \mathrm{~s}$ (including the CPU time for the initialization), respectively. And entropy against itera-

Table 1 Entropy evaluation

\begin{tabular}{ccccc}
\hline & $\mathbf{5 ~} \mathbf{d B}$ & $\mathbf{0 ~ d B}$ & $\mathbf{- 5} \mathbf{~ d B}$ & $\mathbf{- 1 0} \mathbf{~ d B}$ \\
\hline Ideal & 7.170 & 8.169 & 8.813 & 9.155 \\
MERA + MEPA & 7.253 & 8.211 & 8.861 & 9.201 \\
Proposed method & 7.181 & 8.173 & 8.812 & 9.183 \\
\hline
\end{tabular}

tion number in the descent coordinate optimization is shown in the right column of Figure 7. As one can note, the quasi-Newton-based coordinate descent optimization usually achieves convergence within five iterations, taking advantage of the high-accuracy initialization and the usage

Table 2 Contrast evaluation

\begin{tabular}{ccccc}
\hline & $\mathbf{5 ~ d B}$ & $\mathbf{0 ~ d B}$ & $\mathbf{- 5} \mathbf{~ d B}$ & $\mathbf{- 1 0} \mathbf{~ d B}$ \\
\hline Ideal & 0.806 & 0.678 & 0.599 & 0.548 \\
MERA + MEPA & 0.790 & 0.642 & 0.592 & 0.538 \\
Proposed method & 0.805 & 0.677 & 0.599 & 0.544 \\
\hline
\end{tabular}


Table 3 Peak value evaluation

\begin{tabular}{ccccc}
\hline & $\mathbf{5} \mathbf{~ d B}$ & $\mathbf{0 ~ d B}$ & $\mathbf{- 5} \mathbf{d B}$ & $\mathbf{- 1 0 ~ d B}$ \\
\hline Ideal & 43.133 & 45.324 & 43.230 & 43.379 \\
MERA + MEPA & 47.151 & 53.998 & 60.417 & 20.012 \\
Proposed method & 43.130 & 44.725 & 41.384 & 40.719 \\
\hline
\end{tabular}

of second-order derivative. For further comparison of the two corrections, the range profiles from the translational motion compensation under different SNRs are presented in Figure 8. The arrangement of Figure 8 is corresponding to that of Figure 7. What's more, we also use the image entropy, contrast, and peak value to evaluate the focused images in Figure 7, which are presented in Tables 1, 2, and 3 , respectively. It is notable in Figure 7 that due to presence of strong noise, the correction by MERA and MEPA is not precise enough, resulting in blurring and smearing in the RD images. The proposed joint correction method can generate better images after only several iterations (although we pre-set the iteration number as 10 , the joint correction needs only about three iterations to reach the convergence) with the help of high-precision initialization estimation. We also note that both contrast and entropy of images after joint error corrected by the joint correction are very close to those of standard images, which indicates the effectiveness of the method in front of strong noise. In the aspect of computational efficiency, as the coarse motion usually provides estimate with relatively high accuracy, the coordinate descent algorithm then compensates the residual error efficiently. For the Yak- 42 dataset, it is competitive to the minimum entropy-based two-step scheme in efficiency. From Figure 6b, we note two dominant scatters are present in the range bin 61 . We plot this range bin corresponding to different SNRs $(5,0,-5$, and $-10 \mathrm{~dB}$ ) in Figure 9, in order to show the improvement on the point spread function from the use of the joint motion compensation. From Figure 9, we note that the compensation by MERA and MEPA is sensitive to the strong noise, leading to serious distortion and blurring in the result. The range profiles are misaligned under low SNRs, which

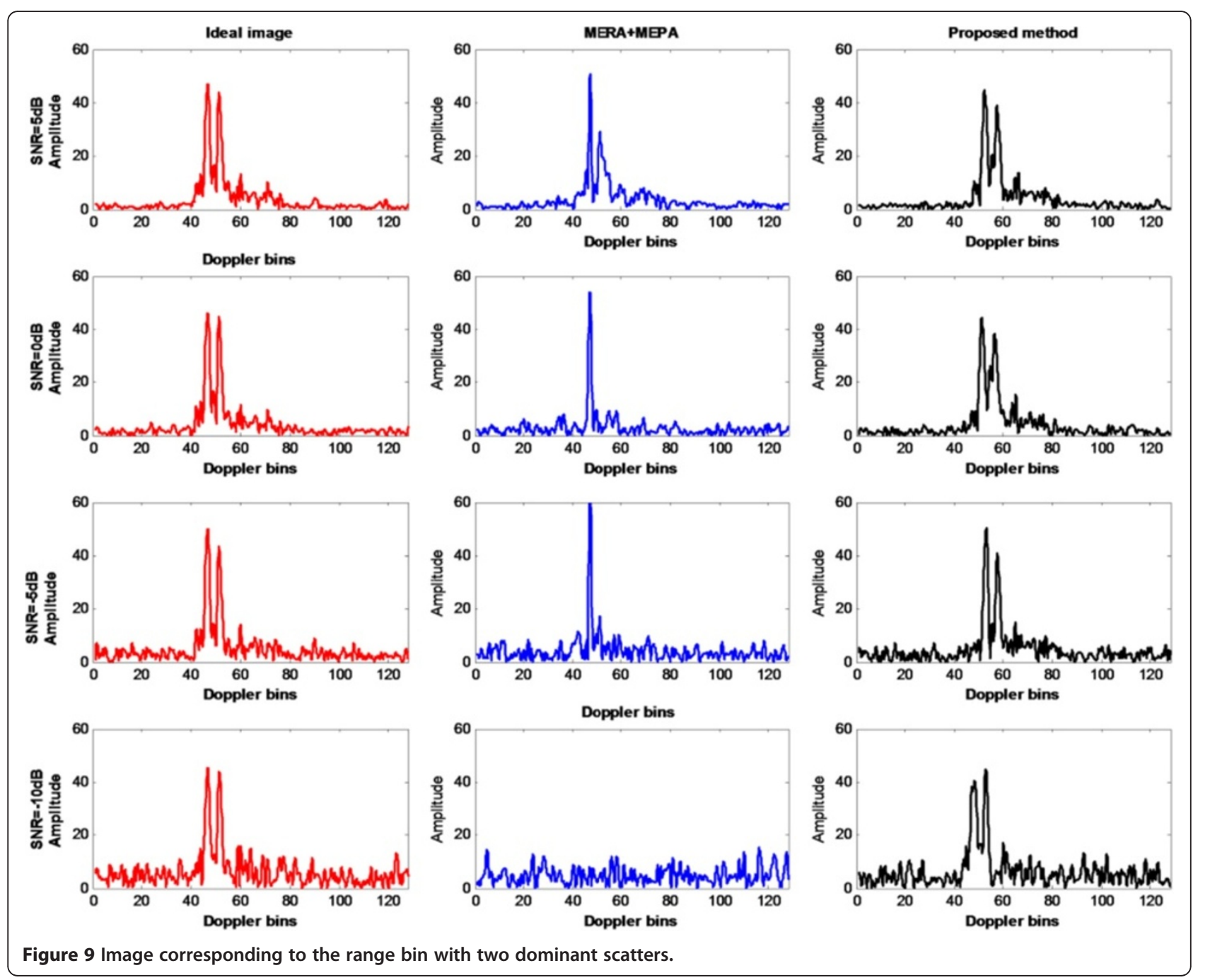




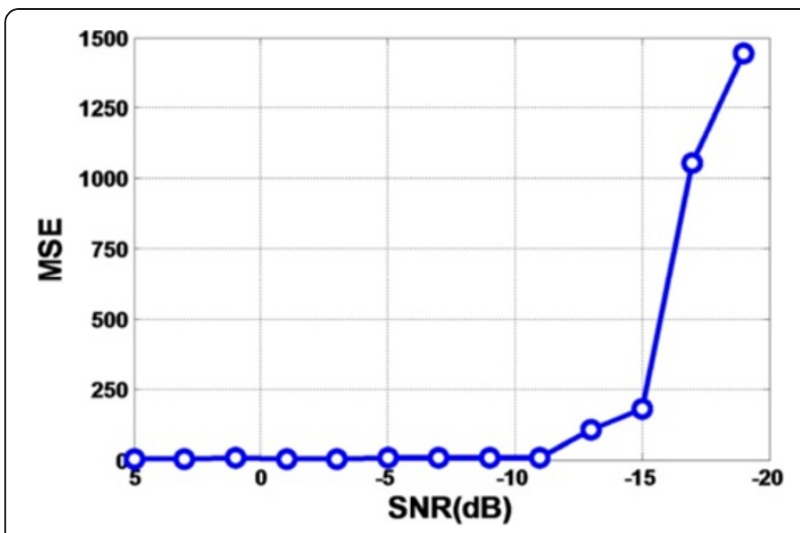

Figure 10 MSE under different SNRs.

can be found from Figure 8 . That is the reason why the peak values of RD image from the two-step compensation become larger than those from the joint correction in Table 2. However, using a parametric motion model, the joint compensation usually removes the motion optimally and the two dominant scatters are separated distinctively. The responses of them are also very close to the standard ones. What's more, as Figure 8 shows, the range profiles from joint correction are also very close to ideal ones. In the experiments, it is found that when the SNR decreases below $-12 \mathrm{~dB}$, both the initialization estimation and the coordinate descent optimization fail to achieve an accurate estimation of the motion error in the joint correction. To show this, the mean square error between the estimated and the real polynomial coefficients is defined by

$$
M S E=\frac{1}{K} \sum_{k=1}^{K}[\alpha(k)-\widetilde{\alpha}(k)]^{2}
$$

The MSEs corresponding to different SNRs are plotted in Figure 10 which presents that the joint correction provides very small MSE only when SNR is above $-12 \mathrm{~dB}$. As mentioned in the last section, in the joint minimum entropy correction, coarse motion estimation for initialization is very essential, as only good initialization can avoid the coordinate descent algorithm being trapped in a local minimum of the entropy optimization. In these experiments, five iterations are used in the initialization estimation and order determination according to the procedure in Figure 4, consuming CUP time of coarse motion estimation corresponding to different SNRs is 1.13, 1.16, 1.86, and 2.17 s, respectively. For clarity, the estimated radial ranges from the coarse motion estimation under different SNRs are presented in Figure 11. From Figure 11, one can note that effective coarse motion estimation is achievable even SNR decreases down to $-10 \mathrm{~dB}$, and the estimate accuracy degrades slightly with the increase of strong noise. For this dataset, the initialized estimates give high accuracy until SNR decreases below $-12 \mathrm{~dB}$, which also leads to failure of the coordinate decent algorithm for fine translational motion correction subsequently as we mentioned before, because the relationship between
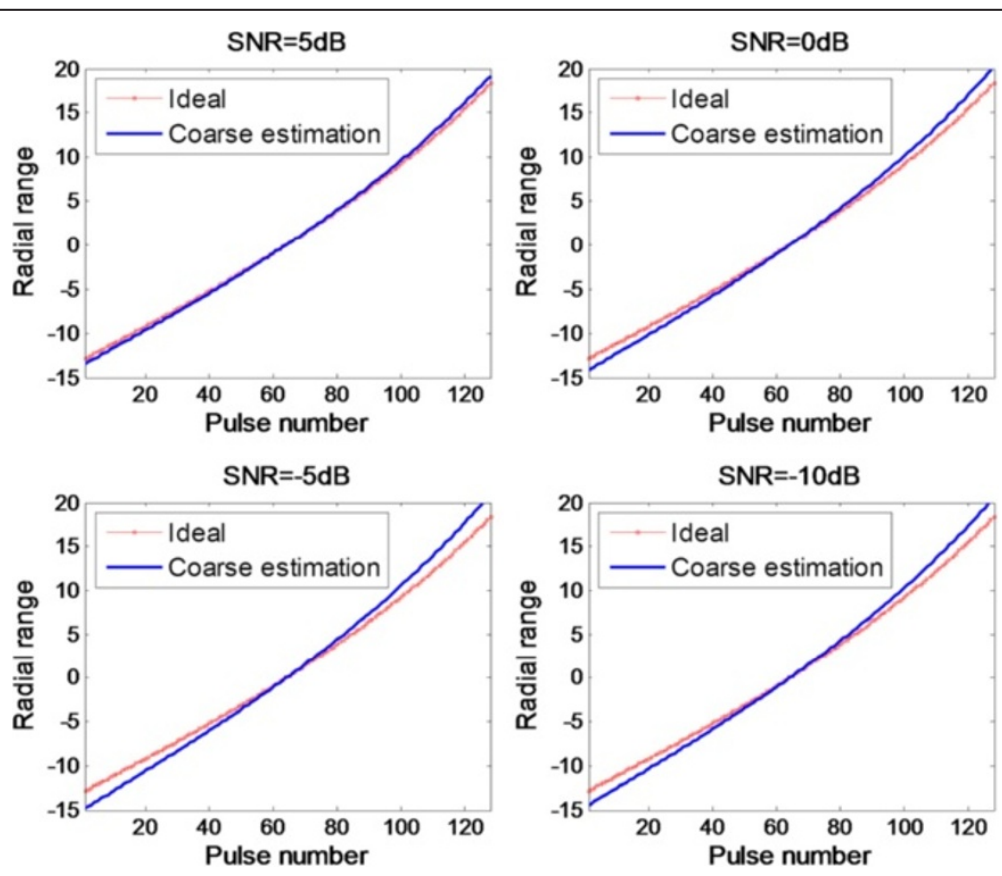

Figure 11 Coarse estimation by the minimum entropy. 


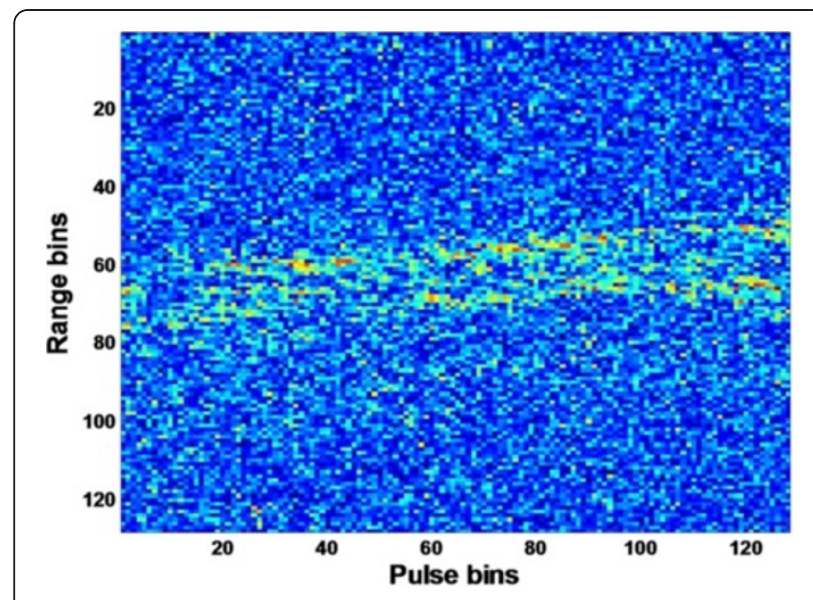

Figure 12 Aligned HRRPs of a ship.

focusing quality and image entropy is inconsistent when very strong noise is involved into the data. And the entropy of image almost relies on the strong noise only, which is independent of the motion correction. In this situation, one can use more pulses to obtain high-SNR gain, and then optimal motion correction and well-focused image may be generated by the proposed method. However, more pulses means longer CPI, which may lead to the 2D rotation model in Figure 1b being not rigid to apply. It should be considered in extremely low SNR scenarios.

The generation of high-resolution ISAR images of ships is usually challenging due to random perturbing rotational motions in pitch, roll, and yaw [34]. Another adverse factor relies on interference from strong clutter noise, which is usually strong enough to degrade the performance of conventional correction methods. Herein, we demonstrate the enhanced performance of joint range alignment and phase adjustment based on minimum entropy to deal with real ship data. The dataset is measured by an airborne synthetic aperture radar system, some radar parameters are listed as follows: the signal carrier frequency is $15 \mathrm{GHz}$ with a bandwidth of $800 \mathrm{MHz}$ to provide range resolution of $0.1875 \mathrm{~m}$ and the pulse repetition frequency is $125 \mathrm{~Hz}$. The numbers of pulse used in the experiment is 128 . Figure 12 shows the HRRPs with motion error. Clearly, due to strong background clutter, the HRRPs are dim. Figure 13a shows the RD image obtained by MERA and MEPA with contrast equivalent to 0.577 , and Figure 13b shows the RD image generated by the joint correction, with larger contrast equivalent to 0.589 at a price of a little computation increase. From this comparison, we believe that improvement is achieved by the joint correction and it is useful in processing the ship ISAR data.

\section{Conclusions}

In this article, we focus on the error correction under low SNR for ISAR imaging. It is widely accepted that minimum entropy-based phase correction is very robust to noise due to the high-SNR gain from the 2D coherent integral in image generation. Inspired by this, a novel scheme is established by using the $2 \mathrm{D}$ image entropy as the penalty function to jointly optimize the range shift and phase error. Implemented by quasi-Newton algorithm, a coordinate descent algorithm is developed to estimate the high-order coefficients of the translational motion. To avoid being trapped into a local minimum far away from the ideal solution, a novel method for coarse motion estimation is also proposed. Coarse motion usually provides solution close to the global optimization, leading to significant iteration number decrease in the fine estimation. Utilizing a parametric model on the range history, the proposed method performs well under strong noise. However, this model restricts the translational motion model, leading to limitations on specific scenarios. For example, severe vibration of the target or radar platform could induces random range shifts and phase errors. In this case, random translational motion compensation for ISAR imaging under low SNR is still open.
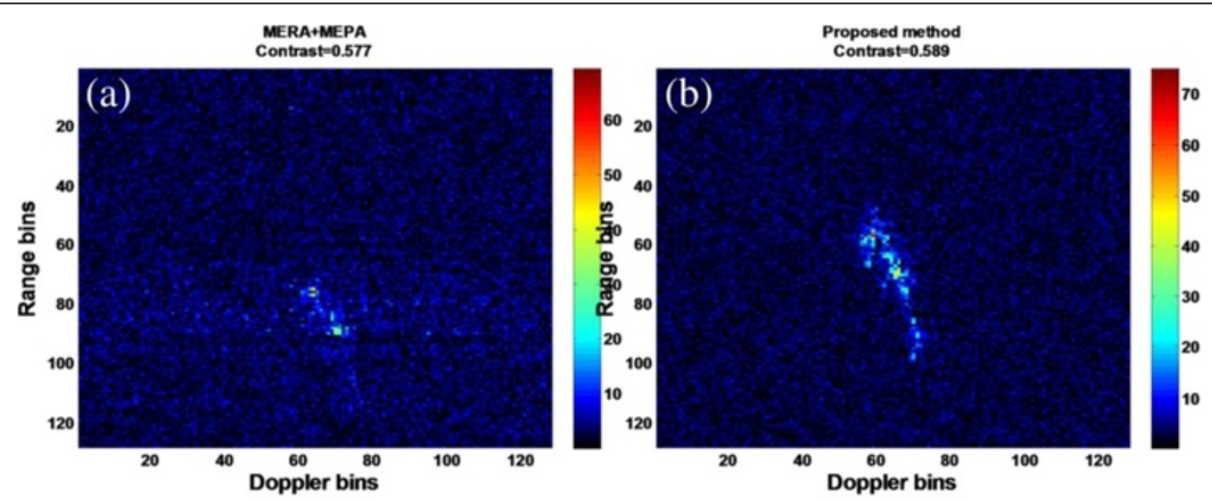

Figure 13 RD image comparison. (a) RD image obtained by conventional minimum entropy methods. (b) RD image obtained after joint range alignment and phase adjustment. 


\section{Appendix}

Based on Equation (34), we have

$$
a\left(\widetilde{\alpha}^{(l, k)} ; \Delta \widetilde{\alpha}_{k}\right)=-\frac{1}{S_{g}} \sum_{k=0}^{M-1} \sum_{h=0}^{N-1}\left[\left(1+\ln \left|g\left(k, h ; \widetilde{\alpha}^{(l, k)} ; \Delta \widetilde{\alpha}_{k}\right)\right|^{2}\right) \cdot \frac{\left.\partial^{2}\left|g\left(k, h ; \widetilde{\alpha}^{(l, k)} ; \Delta \widetilde{\alpha}_{k}\right)\right|^{2}\right]}{\partial \Delta \widetilde{\alpha}_{k} \partial \Delta \widetilde{\alpha}_{k}}\right]
$$

where

$$
\frac{\partial^{2}\left|g\left(k, h ; \widetilde{\alpha}^{(l, k)} ; \Delta \widetilde{\alpha}_{k}\right)\right|^{2}}{\partial \Delta \widetilde{\alpha}_{k} \partial \Delta \widetilde{\alpha}_{k}}=2 \cdot \operatorname{Re}\left[\frac{\partial g^{*}\left(k, h ; \widetilde{\alpha}^{(l, k)} ; \Delta \widetilde{\alpha}_{k}\right)}{\partial \Delta \widetilde{\alpha}_{k}} \cdot \frac{\partial g\left(k, h ; \widetilde{\alpha}^{(l, k)} ; \Delta \widetilde{\alpha}_{k}\right)}{\partial \Delta \widetilde{\alpha}_{k}}+g^{*}\left(k, h ; t_{e} ; \phi\right) \cdot \frac{\partial^{2} g\left(k, h ; t_{e} ; \phi\right)}{\partial \Delta \widetilde{\alpha}_{k} \partial \Delta \widetilde{\alpha}_{k}}\right]
$$

According Equation (36), we have

$$
\begin{aligned}
& \frac{\partial g^{*}\left(k, h ; \widetilde{\alpha}^{(l, k)} ; \Delta \widetilde{\alpha}_{k}\right)}{\partial \Delta \widetilde{\alpha}_{k}}=\left[\frac{\partial g\left(k, h ; \widetilde{\alpha}^{(l, k)} ; \Delta \widetilde{\alpha}_{k}\right)}{\partial \Delta \widetilde{\alpha}_{k}}\right]^{*} \\
& \frac{\partial^{2} g\left(k, h ; t_{e} ; \phi\right)}{\partial \Delta \widetilde{\alpha}_{k} \partial \Delta \widetilde{\alpha}_{k}}=-\frac{16 \pi^{2} \ddot{A}^{N-1}}{N M} \sum_{n=0}^{N} \exp \left(j 2 \pi \frac{h n}{N}\right) \cdot \sum_{m=0}^{M-1} \exp \left(j 2 \pi \frac{k m}{M}\right) \cdot s_{c}\left(m, n ; \widetilde{\alpha}^{(l, k)}\right) \\
& \cdot \exp \left[j 4 \pi \frac{\Delta \widetilde{\alpha}_{k} \cdot(n \cdot \Delta t)^{k}}{c} \cdot\left(m \cdot \Delta f_{r}+f_{c}\right)\right] \cdot(n \cdot \Delta t)^{2 k} \cdot\left(\frac{m \cdot \Delta f_{r}+f_{c}}{c}\right)^{2} \cdot
\end{aligned}
$$

By instituting (36), (45), and (46) into (44), the explicit expression of $a\left(\widetilde{\alpha}^{(l, k)} ; \Delta \widetilde{\alpha}_{k}\right)$ is ready to obtain. And $b\left(\widetilde{\alpha}^{(l, k)} ; \Delta \widetilde{\alpha}_{k}\right)$ is given by

$$
b\left(\widetilde{\alpha}^{(l, k)} ; \Delta \widetilde{\alpha}_{k}\right)=-\frac{1}{S_{g}} \sum_{k=0}^{M-1} \sum_{h=0}^{N-1}\left[\frac{1}{\left|g\left(k, h ; \widetilde{\alpha}^{(l, k)} ; \Delta \widetilde{\alpha}_{k}\right)\right|^{2}} \cdot\left(\frac{\partial\left|g\left(k, h ; \widetilde{\alpha}^{(l, k)} ; \Delta \widetilde{\alpha}_{k}\right)\right|^{2}}{\partial \Delta \widetilde{\alpha}_{k}}\right)^{2}\right]
$$

By instituting Equation (35) into (47), we can obtain the analytic expression of $b\left(\widetilde{\alpha}^{(l, k)} ; \Delta \widetilde{\alpha}_{k}\right)$

\section{Competing interests}

The authors declare that they have no competing interests.

\section{Acknowledgments}

The authors thank the anonymous reviewers for their valuable comments to improve the paper quality. This study was supported by " 973 " program of China under grant 2010CB731903 and National Natural Science Foundation of China under grant JJ0200122201 and 61179010. Lei Zhang's work was also supported by the Fundamental Research Funds for the Central Universities under grant K5051302001 and K5051302038.

\section{Author details}

${ }^{1}$ National Key lab of Radar Signal Processing, Xidian University, Xi'an 710071, P. R. China. ${ }^{2}$ Department of Mathematics, University of Texas-Pan American, Edinburg, TX 78539-2999, USA.

Received: 13 June 2012 Accepted: 8 January 2013 Published: 22 February 2013

\section{References}

1. DL Mensa, High Resolution Radar Imaging (Artech HouseDedham, Dedham, MA, 1981)

2. DR Wehner, High Resolution Radar (Artech House, Norwood, MA, USA, 1995) 3. WC Carrara, RS Goodman, RM Majewsky, Spotlight Synthetic Aperture Radar: Signal Processing Algorithms ((Artech House, Boston, MA, USA, 1995) 
4. CC Chen, HC Andrews, Target-motion-induced radar imaging. IEEE Trans. Aerosp. Electron. Syst. AES-16, 2-14 (1980)

5. T Sauer, A Schroth, Robust range alignment algorithm via Hough transform in an ISAR imaging system. IEEE Trans. Aerosp. Electron. Syst. 31, 1173-1177 (1995)

6. J Wang, D Kasilingam, Global range alignment for ISAR. IEEE Trans. Aerosp. Electron. Syst. 39, 351-357 (2003)

7. JF Wang, XZ Liu, Improved global range alignment for ISAR. IEEE Trans. Aerosp. Electron. Syst. 43, 1070-1075 (2007)

8. D Zhu, L Wang, Y Yu, Q Tao, Z Zhu, Robust ISAR range alignment via minimizing the entropy of the average range profile. IEEE Geosci. Remote Sens. Lett. 6, 204-208 (2009)

9. GY Wang, Z Bao, The minimum entropy criterion of range alignment in ISAR motion compensation, in Proceedings of the IEE Conference on Radar (UK, Edinburgh, 1997), pp. 14-16

10. DE Wahl, PH Eichel, DC Ghiglia, CV Jakowatz Jr, Phase gradient autofocusa robust tool for high resolution SAR phase correction. IEEE Trans. Aerosp. Electron. Syst. 30, 827-834 (1994)

11. Y Wang, H Ling, VC Chen, ISAR motion compensation via adaptive joint time-frequency techniques. IEEE Trans. Aerosp. Electron. Syst. 34, 670-677 (1998)

12. W Ye, TS Yeo, Z Bao, Weighted least-squares estimation of phase errors for SAR/ISAR autofocus. IEEE Trans. Geosci. Remote Sens. 37, 2487-2494 (1999)

13. F Berizzi, G Cosini, Autofocusing of inverse synthetic aperture radar images using contrast optimization. IEEE Trans. Aerosp. Electron. Syst. 32, 1185-1191 (1996)

14. M Martorella, F Berizzi, B Haywood, Contrast maximisation based technique for 2-D ISAR autofocusing. Proc. Inst. Electr. Eng. Radar Sonar Navigat. 152, 253-262 (2005)

15. F Berizzi, M Martorella, A Cacciamano, A Capria, A contrast-based algorithm for synthetic range-profile motion compensation. IEEE Trans. Geosci. Remote Sens. 46, 3053-3062 (2008)

16. F Berizzi, M Martorella, B Haywood, E Dalle Mese, S Bruscoli, A survey on ISAR autofocusing techniques, in Proceedings of the IEEE ICIP 2004 (, Singapore, 2004), pp. 36-41

17. $X L \mathrm{Li}, \mathrm{GS} \mathrm{Liu}, \mathrm{JL} \mathrm{Ni}$, Autofocusing of ISAR images based on entropy minimization. IEEE Trans. Aerosp. Electron. Syst. 35, 1240-1251 (1999)

18. J Wang, X Liu, Z Zhou, Minimum-entropy phase adjustment for ISAR. IEE Proc. Radar Sonar Navigat. 152, 58-67 (2005)

19. J Thomas Kragh, A Alaa Kharbouch, Monotonic iterative algorithm for minimum-entropy autofocus, in IEEE International Conference on Image Processing (, Atlanta, 2006), pp. 645-648

20. J Wang, X Liu, SAR minimum-entropy autofocus using an adaptive order polynomial model. IEEE Geosci. Remote Sens. Lett. 3, 512-516 (2006)

21. H-R Jeong, H-T Kim, K-T Kim, Application of subarray averaging and entropy minimization algorithm to stepped-frequency ISAR autofocus. IEEE Trans. Antennas Propagat. 56, 1144-1154 (2008)

22. J Thomas Kragh, Minimum-entropy autofocus for three-dimensional SAR imaging, in Algorithms for Synthetic Aperture Radar Imagery XVI, ed. by EG Zelnio, FD Garber (SPIE, vol. 7337, 2009), p. 73370B

23. VC Chen, S Qian, Joint time-frequency transform for radar range-Doppler imaging. IEEE Trans. Aerosp. Electron. Syst. 34, 486-499 (1998)

24. T Thayaparan, G Lampropoulos, SK Wong, E Riseborough, Application of adaptive time-frequency algorithm for focusing distorted ISAR images from simulated and measured radar data. IEE Proc. Radar Sonar Navigat. $150,213-220(2003)$

25. JF Li, H Ling, Use of genetic algorithms in ISAR imaging of targets with higher order motions. IEEE Trans. Aerosp. Electron. Syst. 39, 343-351 (2003)

26. IG Cumming. FH Wong, Digital Processing of Synthetic Aperture Radar Data: Algorithms and Implementation ((Artech House, Norwood, MA, 2005)

27. RP Perry, RC Dipietro, RL Fante, SAR imaging of moving targets. IEEE Trans. Aerosp. Electron. Syst. 35, 57-66 (1999)

28. MD Xing, RB Wu, JQ Lan, B Zheng, Migration through resolution cell compensation in ISAR imaging. IEEE Geosci. Remote Sens. Lett. 1, 141-144 (2004)

29. VC Chen, H Ling, Time-Frequency Transforms for Radar Imaging and Signa Analysis (Artech House Inc, Norwood, MA, 2002)

30. AV Oppenheim, AS Willsky, Signal and Systems, 2nd edn. (Prentice Hall, Upper Saddle River, NJ, 1997)
31. X Guo, HB Sun, TS Yeo, Transient interference excision in over-the-horizon radar using adaptive time-frequency analysis. IEEE Trans. Geosci. Remote Sens. 43, 722-735 (2005)

32. M Martorella, F Berizzi, S Bruscoli, Use of genetic algorithms for contrast maximization and entropy minimization in ISAR autofocusing. J. Appl. Signal Process 2006, 1-11 (2006). Special Issue on "Inverse Synthetic Aperture Radar"

33. SB Peng, J Xu, YN Peng, JB Xiang, Parametric inverse synthetic aperture radar maneuvering target motion compensation based on particle swarm optimizer. IET Radar Sonar Navigat. 5, 305-314 (2011)

34. D Pastina, A Montanari, A Aprile, Motion estimation and optimum time selection for ship ISAR imaging, in Proceedings of the Radar Conference (AL, Huntsville, 2003), pp. 7-14

doi:10.1186/1687-6180-2013-33

Cite this article as: Zhang et al: Translational motion compensation for ISAR imaging under low SNR by minimum entropy. EURASIP Journal on Advances in Signal Processing 2013 2013:33.

\section{Submit your manuscript to a SpringerOpen ${ }^{\circ}$ journal and benefit from:}

- Convenient online submission

- Rigorous peer review

- Immediate publication on acceptance

- Open access: articles freely available online

- High visibility within the field

- Retaining the copyright to your article

Submit your next manuscript at $>$ springeropen.com 